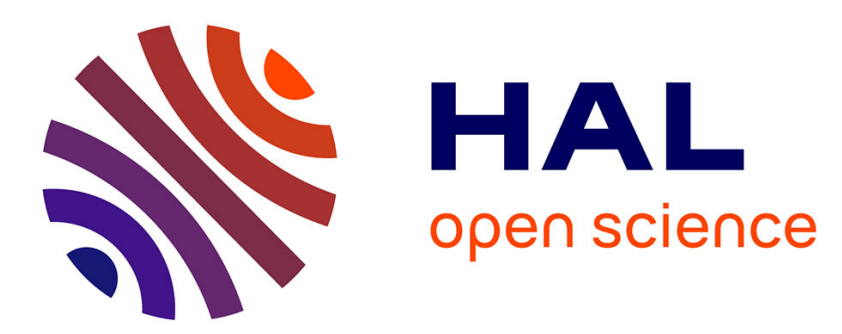

\title{
Design and Comparison of Lattices of Topological Relations for Spatial Representation and Reasoning
}

Florence Le Ber, Ludmila Mangelinck, Amedeo Napoli

\section{To cite this version:}

Florence Le Ber, Ludmila Mangelinck, Amedeo Napoli. Design and Comparison of Lattices of Topological Relations for Spatial Representation and Reasoning. [Research Report] RR-4321, INRIA. 2001, pp.57. inria-00072266

\section{HAL Id: inria-00072266 \\ https://hal.inria.fr/inria-00072266}

Submitted on 23 May 2006

HAL is a multi-disciplinary open access archive for the deposit and dissemination of scientific research documents, whether they are published or not. The documents may come from teaching and research institutions in France or abroad, or from public or private research centers.
L'archive ouverte pluridisciplinaire HAL, est destinée au dépôt et à la diffusion de documents scientifiques de niveau recherche, publiés ou non, émanant des établissements d'enseignement et de recherche français ou étrangers, des laboratoires publics ou privés. 
INSTITUT NATIONAL DE RECHERCHE EN INFORMATIQUE ET EN AUTOMATIQUE

\section{Design and comparison of lattices of topological relations for spatial representation and reasoning}

Florence Le Ber — Ludmila Mangelinck — Amedeo Napoli

$$
\mathbf{N}^{\circ} 4321
$$

Novembre 2001

THÈME 3 



\title{
Design and comparison of lattices of topological relations for spatial representation and reasoning
}

\author{
Florence Le Bet田, Ludmila Mangelinck 团, Amedeo Napol目 \\ Thème 3 - Interaction homme-machine, \\ images, données, connaissances \\ Projet Orpailleur \\ Rapport de recherche $\mathrm{n}^{\circ} 4321$ - Novembre 2001 - 57 pages
}

\begin{abstract}
This report presents an original approach to spatial representation and reasoning with topological relations based on the use of lattices of relations. This approach has been developed for spatial reasoning in the domain of agricultural landscape analysis. The report describes first the motivation of the present research work and the general framework of topological relations. Four different lattices of topological relations, including Galois lattices, are introduced. The choices made for spatial representation and reasoning with these lattices are discussed, together with a thorough study and comparison of the four lattices. The implementation of one of the lattices within an object-based representation system, as well as spatial lattice-based reasoning with topological relations in this system are then detailed.
\end{abstract}

Key-words: Topological relations, lattice-based classification, Galois connection, relation computation, raster images, object-based representation systems

* LORIA INRIA

$\dagger$ Thèse financée par l'INRA et la Région Lorraine

$\ddagger$ LORIA CNRS 


\section{Définition et comparaison de treillis de relations topologiques pour la représentation et le raisonnement sur l'espace}

Résumé : Ce rapport présente une approche originale pour la représentation de l'espace et le raisonnement spatial qualitatif. Cette approche s'appuie sur l'utilisation de treillis de relations topologiques et a été développée à des fins de reconnaissance d'organisations spatiales de paysages dans le domaine agronomique. Le rapport décrit d'abord la motivation de ce travail et le cadre théorique des relations topologiques dans lequel nous nous plaçons. Nous présentons quatre treillis de relations topologiques, dont deux sont des treillis de Galois. Nous discutons et comparons les capacités de ces treillis en matière de représentation et de raisonnement. Finalement, nous décrivons l'implantation et l'utilisation d'un de ces treillis pour la classification de structures spatiales dans le cadre d'un système de représentation de connaissances par objets.

Mots-clés : Relations topologiques, classification à partir de treillis, Correspondance de Galois, calcul de relations, images rasteur, représentation de connaissances par objets 


\section{Introduction}

The study of land use is at present of first importance in agronomics, in order to understand the reorganization of farmland, the transformations of landscape and the alterations of the environment, depending on changes of the agriculture, e.g. enlargement of farm enterprises, modification of agricultural practices (cropping plans, etc.). This study leads to a number of qualitative and quantitative questions regarding the interpretation of present and past land use, the prediction of land use in the near future, the influences of the agricultural practices on the structure of land use, the conditions that have to be fulfilled to reach a desired landscape, etc. Moreover, it could be possible to understand the present state and the near future of land use and agriculture on the basis of the present observations.

Actually, these questions are linked together in the agronomics researches about agricultural landscape analysis, that is carried on the basis of field inquiries and spatial observations on maps, aerial photographs and satellite images. This is one of the fundamental reasons why landscape analysis is of main importance in the agronomics researches nowadays [Deffontaines 1986. For that purpose, the agronomics researchers are particularly interested in the relationship between landscape and farming systems and their consequences on the environment. They try to discover and formalize landscape patterns that are particular structures of land use, which are linked to specific combinations of factors (soil, relief, settlement, farming system) [Deffontaines 1990, Benoît 1990. Agronomics researchers assume that the structure of land use reveals the working of the farming system. Landscape patterns exist at several scales and are very useful for global diagnoses and forecasts on agriculture with regard to the environment.

Land-use maps based on satellite images are very useful for landscape analysis. However because of the quantity and frequency of these data, an automatic processing is needed. To be more efficient such processing must include the expertise of the researchers about farming systems and land use. Thus, it is worthwhile to use AI methods to build a knowledge-based system that could help agronomists to exploit land-use maps for landscape analysis. Therefore, the landscape patterns defined by the agronomics researchers have been adapted for classifying zones extracted on the maps Le Ber and Mangelinck 1998. Each zone corresponds to a "village territory" which is supposed to be exploited by the farmers of this village. A zone is made of raster regions, i.e. connected sets of pixels with the same label denoting the land-use category, e.g. crops, meadows, forest, village, etc. The adapted landscape patterns, called landscape models, are described with a list of agricultural spatial entities and a set of qualitative spatial relations between these entities.

From an implementation point of view, an object-based knowledge representation system, or OKR system, equipped with classification methods has been used [Napoli 1992. Napoli et al.1994. Indeed, exploiting land-use maps for landscape analysis may be considered as an instance classification problem, where landscape models correspond to classes, and zones correspond to instances that have to be classified according to landscape models. For solving such a classification problem, the landscape models have to be represented, implying that knowledge on agricultural entities and on spatial relations must be encoded. Besides, methods for extracting regions from the land-use maps and for checking spatial re- 
Le Ber, Mangelinck \& Napoli

lations on these regions have been defined Mangelinck et al.1996. These methods are used as a basis for a classification process, whose goal is to compare the regions and the relations extracted from a land-use map under study to the entities and the relations associated with the landscape models.

In our application, the classification process is mainly based on the spatial relations, since they are the most characteristic elements of the landscape models. We have focused on the representation of topological relations because they can be described and manipulated on the basis of a well defined theoretical framework. Relying on this framework, we need a representation system of the topological relations that:

- defines primitives to compute relations on raster images: to compute -or to check- a relation $R$ between two regions $x$ and $y$ means to test if $R(x, y)$ holds.

- integrates all the relations used in the landscape models,

- allows to store new information,

- allows reasoning on relations and composition of relations: to reason on relations means to deduce new relations from already computed ones.

- can be managed by an OKR system, and thus, the application must be low-sized in terms of memory space.

According to these needs, we have designed a representation of the topological relations based on a lattice structure. Such a representation has been already used for example in Randell and Cohn 1992] to draw inferences on relations. We have built four lattices, including two lattices based on the Galois connection, that is used to create concepts, by associating sets of objects (the extents of the concepts) to sets of attributes (the intents) Barbut and Monjardet 1970, Davey and Priestley 1990, Wille 1992. In this way, relations that have to be recognized on an image can be associated to properties that can be computed on the image. The different lattices have been evaluated and implemented in the framework of our application.

The work presented here shows several new and original aspects in the management of spatial relations, regarding representation, reasoning and implementation aspects. For the representation and reasoning purposes, the theory of Galois lattices has been employed with success and has allowed to build different lattices providing different points of view on the topological relations, depending on the way the boundary of a raster region is considered. Moreover, it is one of the unique works relying on the Galois lattice theory, that is usually associated with formal concept analysis [Wille 1992, or for knowledge discovery purposes Godin and Missaoui 1994, Simon and Napoli 1999. Finally, classification-based reasoning is used for inferring spatial relations and spatial structures of agricultural entities, involving both classification of entities and relations. Another noticeable aspect is that this work allows a qualitative as well as a quantitative approach to the analysis of landscape on landuse maps, because the chosen implementation framework, i.e. OKR systems, provides both reasoning and computation facilities. This contrasts with existing works in the domain of 
qualitative spatial reasoning, which mainly focus on reasoning about qualitative relations only. Indeed, our system combines symbolic and numerical techniques for reasoning about spatial entities and their qualitative relations and provides results that are easy to read and understand for agronomists. This research work can be naturally extended to the design of geographic information systems.

This article describes our work on the definition and the comparison of the different lattices of topological relations. It is structured as follows: the first part introduces the context of our work and describes the spatial theories on which it is based; the second part presents our proposition for computing topological relations on raster images; the third and fourth parts describe the various possibilities that have been explored for building lattices and a comparison of these lattices. The fifth part presents some elements about the implementation of the relations and their use for the classification of spatial structures. The sixth part gives an evaluation and some perspectives of the present research work. Finally we conclude.

\section{Related work}

In the section we present research works that are related to our work and that, for some of them, have inspired our work.

\subsection{Topological relations: theories}

Mathematical topology is based on the "open set" notion and defines the notions of "closed set" and "neighborhood". Topology can also be defined with the notion of continuity: two forms are topologically equivalent if one form can be continuously transformed (i.e. with no hole) into the other: for instance, an egg is equivalent to a ball but is not equivalent to a torus. Topology can then be used to characterize forms.

In qualitative representations of space, topological relations are opposed to distance and orientation relations. Topological relations are binary and independent of the orientation of the entities. The basic ontological entity is generally a region. Regions represent portions of space determined by concrete objects, and are not considered as sets of spatial points Vieu 1993.

Mereology is a theory of the part-whole relation. It was introduced by Lesniewski at the beginning of 20th century as an alternative to the set theory [Lesniewski 1927 1931]. It is an axiomatic base for topology and geometry of regions rather than of points. Mereology defines the relation $P(x, y)$, for " $x$ is a part of $y$ ", which is a partial order since it is reflexive, anti-symmetric and transitive:

$$
\begin{array}{lll}
\forall x \in \mathcal{D} & : & P(x, x) \\
\forall x, y \in \mathcal{D} & : & (P(x, y) \wedge P(y, x)) \rightarrow x=y \\
\forall x, y, z \in \mathcal{D} & : & (P(x, y) \wedge P(y, z)) \rightarrow P(x, z)
\end{array}
$$


Le Ber, Mangelinck \& Napoli

Seven relations can be defined from this primitive relation (see Table 1 ). The $P P(x, y)$ relation means " $x$ is a proper part of $y$ ". The $O(x, y)$ relation means " $x$ overlaps $y$ ". The $U(x, y)$ relation means " $x$ and $y$ are part of the same region". The remaining relations represent various cases of the "overlap" and "inclusion" notions.

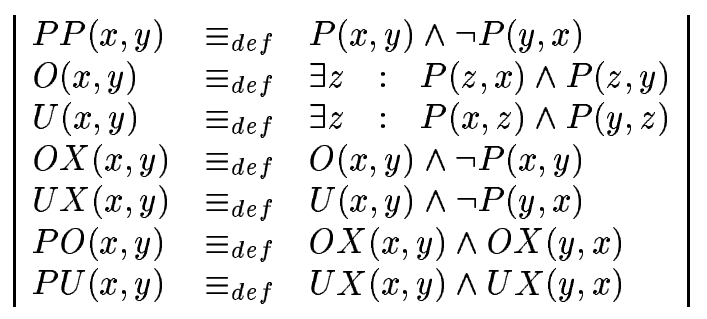

Table 1: Mereological relations (see [Vieu 1993, Varzi 1996]). The symbols $\wedge$ and $\neg$ represent the usual logical connectives.

Clarke has later proposed to formalize the part-whole relation using the "connection" notion Clarke 1981, Clarke 1985. Two regions $x$ and $y$ are said to be connected $-C(x, y)-$ if they share a point. A point is a zero-dimensional spatial element, according to its mathematical meaning. The Clarke's theory is defined by the following axioms:

$$
\begin{array}{lll}
\forall x \in \mathcal{D} & : & C(x, x) \\
\forall x, y \in \mathcal{D} & : \quad C(x, y) \rightarrow C(y, x) \\
\forall x, y \in \mathcal{D} & : \quad(\forall z \in \mathcal{D}: C(z, x) \leftrightarrow C(z, y)) \rightarrow x=y
\end{array}
$$

Relying on the connection relation, Clarke has defined five relations: $D C(x, y)$ " $x$ is disconnected from $y$ "; $P(x, y)$ " $x$ is a part of $y$ "; $P P(x, y)$ " $x$ is a proper part of $y$ "; $O(x, y)$ " $x$ overlaps $y$ "; $D R(x, y)$ " $x$ is discrete from $y$ ". The theory based on these relations is interesting because it makes a difference between a point and a region, thanks to the relations $C(x, y)-x$ and $y$ share a point- and $O(x, y)-x$ and $y$ share a region. This differentiation allows the definition of three additional relations: $E C(x, y)$ " $x$ is externally connected with $y$ ", $T P(x, y)$ " $x$ is a tangential part of $y$ ", $N T P(x, y)$ " $x$ is a non tangential part of $y$ " (see Table 2). Finally this differentiation is used to introduce topological notions (interior, boundary) into the purely set-based notions of the mereology: the Clarke's theory is therefore called mereotopology.

Several theories have then been proposed for topological relations [Randell et al. 1992b, Vieu 1993. These theories are generally formalized in first order logic. The differences between the theories are about the number of relations, and the interpretation of the relations. For instance, Randell et al.1992a] defines a theory with 10 relations including the "internal part" notion whereas [Cohn et al.1993] defines a theory with 16 relations including relations based on the "convex hull" notion: OUTSIDE, INSIDE, etc. Each theory is defined by a set of axioms, depending on the desired properties of the spatial regions (open 


$$
\left|\begin{array}{lll}
D C(x, y) & \equiv_{\text {def }} & \neg C(x, y) \\
P(x, y) & \equiv_{\text {def }} & \forall z \quad: \quad C(z, x) \rightarrow C(z, y) \\
P P(x, y) & \equiv_{\text {def }} & P(x, y) \wedge \neg P(y, x) \\
O(x, y) & \equiv_{\text {def }} & \exists z \quad: \quad P(z, x) \wedge P(z, y) \\
D R(x, y) & \equiv_{\text {def }} & \neg O(x, y) \\
E C(x, y) & \equiv_{\text {def }} & C(x, y) \wedge \neg O(x, y) \\
T P(x, y) & \equiv_{\text {def }} & P(x, y) \wedge \exists z \quad: \quad E C(z, x) \wedge E C(z, y) \\
N T P(x, y) & \equiv_{\text {def }} & P(x, y) \wedge \neg \exists z \quad: \quad E C(z, x) \wedge E C(z, y)
\end{array}\right|
$$

Table 2: Mereotopological relations [Clarke 1981]. The relations $P(x, y), P P(x, y)$, and $O(x, y)$ are identical to the relations $P(x, y), P P(x, y)$, and $O(x, y)$ of mereology (Table 1 .

and closed regions, atomicity, non-atomicity, etc., see [Varzi 1996, Vieu 1997, Varzi 1998, Masolo and Vieu 1999, Masolo 2000 for surveys and discussions on these points of view).

The most used and well-known topological relations are those of the so-called $R C C-8$ theory [Randell et al. 1992b, Cohn et al. 1997] which is also based on the connection relation. This theory defines eight base relations which are exhaustive and mutually exclusive. Their names and iconic representations are given in Table 3 and their definitions in Table 4 .

\begin{tabular}{|c|c|c|}
\hline relation & notation & icons \\
\hline " $x$ is identical with $y "$ & $E Q(x, y)$ & \\
\hline " $x$ is a non tangential proper part of $y "$ & $N T P P(x, y)$ & (1) \\
\hline " $x$ is a tangential proper part of $y "$ & $T P P(x, y)$ & (10) \\
\hline " $x$ non tangentially contains as a proper part $y "$ & $N T P P^{-1}(x, y)$ & 0 \\
\hline " $x$ tangentially contains as a proper part $y "$ & $T P P^{-1}(x, y)$ & 0 \\
\hline "x partially overlaps $y "$ & $P O(x, y)$ & \\
\hline " $x$ is externally connected with $y "$ & $E C(x, y)$ & \\
\hline " $x$ is disconnected from $y "$ & $D C(x, y)$ & (1) \\
\hline
\end{tabular}

Table 3: Names and icons associated to the eight base relations of the $R C C-8$ theory.

\subsection{Computation of topological relations}

In order to deal with applications, such as geographic databases or image interpretation, one needs to compute relations, i.e. one needs operations for testing if a relation holds between two regions. Such computation operations have been proposed in Egenhofer 1989. 


$$
\left|\begin{array}{lll}
E Q(x, y) & \equiv_{\text {def }} & P(x, y) \wedge P(y, x) \\
N T P P(x, y) & \equiv_{\text {def }} & P P(x, y) \wedge \neg \exists z \quad: \quad E C(z, x) \wedge E C(z, y) \\
T P P(x, y) & \equiv_{\text {def }} & P P(x, y) \wedge \exists z \quad: \quad E C(z, x) \wedge E C(z, y) \\
N T P P^{-1}(x, y) & \equiv_{\text {def }} & N T P P(y, x) \\
T P P^{-1}(x, y) & \equiv_{\text {def }} & T P P(y, x) \\
P O(x, y) & \equiv_{\text {def }} & O(x, y) \wedge \neg P(x, y) \wedge \neg P(y, x) \\
E C(x, y) & \equiv_{\text {def }} & C(x, y) \wedge \neg O(x, y) \\
D C(x, y) & \equiv_{\text {def }} & \neg C(x, y)
\end{array}\right|
$$

Table 4: The base relations of the $R C C-8$ theory [Randell et al. 1992b]. Their definitions rely on the mereotopology.

Egenhofer and Sharma 1993, Clementini et al. 1993: they are based on the notions of interior and boundary of a region. The are linked to formal models of the topological relations.

In Egenhofer 1989, computation operations are defined for the vector representation of geographic information systems: a $n$-dimensional region $x$ is characterized by two sets, the interior (of dimension $n$ ), denoted by $x^{\circ}$, and the boundary (of dimension $n-1$ ), denoted by $\partial x$. Two regions are thus characterized by four sets whose intersections define four operations: $\partial x \cap \partial y, x^{\circ} \cap y^{\circ}, \partial x \cap y^{\circ}$ et $x^{\circ} \cap \partial y$. Figure1 and Table[5 show the correspondence between the results of these operations and the eight relations of the $R C C-8$ theory. For instance if the relation " $x$ partially overlaps $y$ ", $P O(x, y)$, holds between the two regions $x$ and $y$, then the four operations applied to $x$ and $y$ give the following results: $\partial x \cap \partial y \neq \emptyset$, $x^{\circ} \cap y^{\circ} \neq \emptyset, \partial x \cap y^{\circ} \neq \emptyset$ and $x^{\circ} \cap \partial y \neq \emptyset$. Conversely, if the four operations give the previous results when applied to the two regions $x$ and $y$, then the relation $P O(x, y)$ holds (Figure 1).

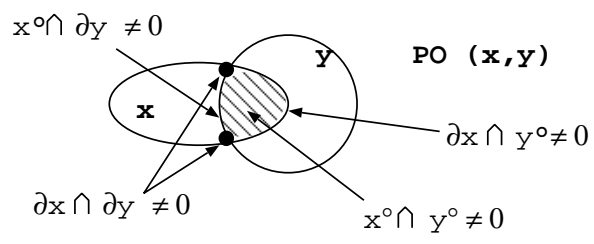

Figure 1: Correspondence between the operations defined in Egenhofer 1989 and the relations of the $R C C-8$ theory: the relation $P O(x, y)$.

The computation method based on these four operations is interesting since it associates the base relations of the $R C C-8$ theory to a set of necessary and sufficient conditions to be checked on the regions. But the regions have to be characterized with an interior and a boundary, which can be difficult to define on raster images, as it is shown below (Section 2.1). 


\begin{tabular}{|l|c|c|c|c|}
\hline $\mathrm{x}, \mathrm{y}$ & $\partial x \cap \partial y$ & $x^{\circ} \cap y^{\circ}$ & $\partial x \cap y^{\circ}$ & $x^{\circ} \cap \partial y$ \\
\hline$E Q(x, y)$ & $\neq \emptyset$ & $\neq \emptyset$ & $\emptyset$ & $\emptyset$ \\
$N T P P(x, y)$ & $\emptyset$ & $\neq \emptyset$ & $\neq \emptyset$ & $\emptyset$ \\
$T P P(x, y)$ & $\neq \emptyset$ & $\neq \emptyset$ & $\neq \emptyset$ & $\emptyset$ \\
$N T P P^{-1}(x, y)$ & $\emptyset$ & $\neq \emptyset$ & $\emptyset$ & $\neq \emptyset$ \\
$T P P^{-1}(x, y)$ & $\neq \emptyset$ & $\neq \emptyset$ & $\emptyset$ & $\neq \emptyset$ \\
$P O(x, y)$ & $\neq \emptyset$ & $\neq \emptyset$ & $\neq \emptyset$ & $\neq \emptyset$ \\
$E C(x, y)$ & $\neq \emptyset$ & $\emptyset$ & $\emptyset$ & $\emptyset$ \\
$D C(x, y)$ & $\emptyset$ & $\emptyset$ & $\emptyset$ & $\emptyset$ \\
\hline
\end{tabular}

Table 5: Correspondence between the relations of the $R C C-8$ theory and the operations on interior and boundary sets proposed in Egenhofer 1989.

\subsection{Mathematical properties of topological relations}

It is often useful to deduce new information from a partial knowledge about a situation or to verify the consistency of the available information in a given situation. This can be done thanks to the following mathematical properties of topological relations, where $\mathcal{D}$ is a set of regions:

- Conversion: the converse relation of a relation $R$ is the relation $R^{-1}$ such that:

$$
\forall(x, y) \in \mathcal{D}^{2}, R(x, y) \leftrightarrow R^{-1}(y, x)
$$

- Disjoint: two relations $R_{1}$ et $R_{2}$ are said to be disjoint if:

$$
\forall(x, y) \in \mathcal{D}^{2}, R_{1}(x, y) \leftrightarrow \neg R_{2}(x, y)
$$

- Complement: the complement of a relation $R$ is the relation $R^{c}$ such that:

$R$ and $R^{c}$ are disjoint and $\forall(x, y) \in \mathcal{D}^{2}, R(x, y) \vee R^{c}(x, y)$ holds.

- Composition: given three regions $x, y, z$ and a pair of relations $R_{1}, R_{2}$ such that $R_{1}(x, y)$ and $R_{2}(y, z)$, the composition of $R_{1}$ and $R_{2}$ is the disjunction $R$ of all the possible relations holding between $x$ and $z$ :

$$
\begin{aligned}
& \forall(x, y, z) \in \mathcal{D}^{3}, R_{1}(x, y) \wedge R_{2}(y, z) \rightarrow R(x, z) \text { and, } \\
& \forall R_{3},\left(R_{1}(x, y) \wedge R_{2}(y, z) \wedge R_{3}(x, z)\right) \rightarrow\left(\forall(u, v) \in \mathcal{D}^{2}, R_{3}(u, v) \rightarrow R(u, v)\right) .
\end{aligned}
$$

The last property is especially interesting: as proposed in [Randell and Cohn 1992, rules of composition are used to deduce the relation that holds between two regions $x$ and $z$ from the knowledge of the relations holding between those two regions and a third region $y$. For instance, Figure 2 depicts two configurations where three regions $x, y$ and $z$ are drawn with respect to the relations $T P P(x, y)$ and $E C(y, z)$. Thus $D C(x, z)$ or $E C(x, z)$ can be deduced. The two schemas of the figure correspond to the two possible relative locations of $x$ and $z$.

The rules of composition on the topological relations are represented in composition tables. Such a table has been used first by Allen Allen 1983 to deduce temporal relations 

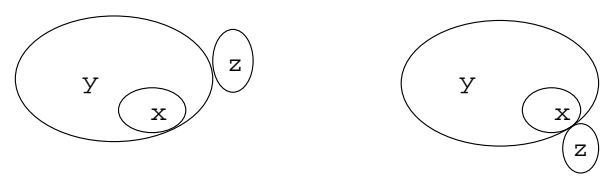

Figure 2: Composition of topological relations. The two configurations are described by $T P P(x, y)$ and $E C(y, z)$. In the left case, $D C(x, z)$ is true, and in the right case, $E C(x, z)$ is true.

between events. Composition tables have also been used in the connection-based theories: such a table is defined for 9 relations in [Randell et al.1992a, and for 22 relations in Cohn et al. 1993 .

A composition table is defined for a subset of disjoint relations of a theory. Table 6 presents the composition table of the eight base relations of the $R C C$-8 theory. The $E Q(x, y)$ " $x$ is identical with $y$ " relation is not represented because it is a neutral element: for any relation $R, R(x, y)$ and $E Q(y, z)$ implies $R(x, z) ; E Q(x, y)$ and $R(y, z)$ implies $R(x, z)$.

\subsection{Some applications in connection with topological relations}

Mereotopological formalisms have been used in various applications.

In [Kawashima et al.1994, topological relations have been used to describe the behavior of groups of soccer players: groups of players are extracted from image sequences, their position is described thanks to the relations of the $R C C-8$ theory. Behaviors are defined according to the possible transitions between the relations. It has been recently suggested to continue this research, in order to analyze and comment robots playing soccer [Nebel 2000].

Clarke's mereotopology has been extended to represent spatial expressions of natural language [Vieu 1993. The part-whole relation, in particular, is used in several ways in natural language [Winston et al. 1987]: for instance, one should distinguish the expressions "the egg is in the bowl" and "the yolk is in the egg". Various notions of inside are defined in [Cohn et al.1997. Furthermore, relying on the topological notions of open and closed sets, different notions of contact can also be defined: external connection $(E C(x, y))$ between two closed regions, contact between a closed region and an open one, weak contact between two open regions [Asher and Vieu 1995].

Computer-aided design systems and geographic information systems also use topological relations. Actually they use both numerical representations (based on the Euclidean space) and qualitative representations (distance, orientation, topology) to meet the users' requirements Cui et al. 1993. Frank 1997. Furthermore, studies are done for applying spatioterminological reasoning in the context of visual-query systems [Möller and Wessel 1999. 


\begin{tabular}{|c|c|c|c|c|c|c|c|}
\hline $\begin{array}{l}\mathrm{R}_{2}(\mathrm{y}, \mathrm{z}) \\
\mathrm{R}_{1}(\mathrm{x}, \mathrm{y})\end{array}$ & NTPP & $\mathrm{TPP}$ & $\mathrm{NTPP}^{-1}$ & $\mathrm{TPP}^{-1}$ & $\mathrm{PO}$ & $\mathrm{EC}$ & $\mathrm{DC}$ \\
\hline NTPP & NTTP & NTPP & T & $\begin{array}{l}\mathrm{DC}, \mathrm{EC}, \\
\mathrm{PO}, \mathrm{TPP}, \\
\mathrm{NTPP}\end{array}$ & $\begin{array}{l}\text { DC, EC, } \\
\text { PO, TPP, } \\
\text { NTPP }\end{array}$ & DC & $\mathrm{DC}$ \\
\hline $\mathrm{TPP}$ & NTPP & $\begin{array}{l}\text { TPP, } \\
\text { NTPP }\end{array}$ & $\begin{array}{l}\mathrm{DC}, \\
\mathrm{EC}, \mathrm{PO}, \\
\mathrm{TPP}^{-1}, \\
\mathrm{NTPP}^{-1}\end{array}$ & $\begin{array}{l}\mathrm{DC}, \mathrm{EC}, \\
\mathrm{PO}, \mathrm{TPP}, \\
\mathrm{TPP}^{-1}, \\
\mathrm{EQ}\end{array}$ & $\begin{array}{l}\text { DC, EC, } \\
\text { PO, TPP, } \\
\text { NTPP }\end{array}$ & $\mathrm{DC}, \mathrm{EC}$ & $\mathrm{DC}$ \\
\hline $\mathrm{NTPP}^{-1}$ & $\begin{array}{l}\text { PO, TPP, } \\
\text { NTPP, } \\
\text { TPP }^{-1}, \\
\text { NTPP }^{-1}, \\
\text { EQ }\end{array}$ & $\begin{array}{l}\mathrm{PO}, \\
\mathrm{TPP}^{-1}, \\
\mathrm{NTPP}^{-1}\end{array}$ & $\mathrm{NTPP}^{-1}$ & $\mathrm{NTPP}^{-1}$ & $\begin{array}{l}\mathrm{PO}, \\
\mathrm{TPP}^{-1}, \\
\mathrm{NTPP}^{-1}\end{array}$ & $\begin{array}{l}\mathrm{PO}, \\
\mathrm{TPP}^{-1}, \\
\mathrm{NTPP}^{-1}\end{array}$ & $\begin{array}{l}\mathrm{DC}, \\
\mathrm{EC}, \mathrm{PO}, \\
\mathrm{TPP}^{-1}, \\
\mathrm{NTPP}^{-1}\end{array}$ \\
\hline $\mathrm{TPP}^{-1}$ & $\begin{array}{l}\text { PO, TPP, } \\
\text { NTPP }\end{array}$ & $\begin{array}{l}\mathrm{PO}, \mathrm{TPP}, \\
\mathrm{TPP}^{-1}, \\
\mathrm{EQ}\end{array}$ & $\mathrm{NTPP}^{-1}$ & $\begin{array}{l}\mathrm{TPP}^{-1}, \\
\mathrm{NTPP}^{-1}\end{array}$ & $\begin{array}{l}\mathrm{PO}, \\
\mathrm{TPP}^{-1}, \\
\mathrm{NTPP}^{-1}\end{array}$ & $\begin{array}{l}\mathrm{EC}, \quad \mathrm{PO}, \\
\mathrm{TPP}^{-1}, \\
\mathrm{NTPP}^{-1}\end{array}$ & $\begin{array}{l}\mathrm{DC}, \\
\mathrm{EC}, \mathrm{PO}, \\
\mathrm{TPP}^{-1}, \\
\mathrm{NTPP}^{-1}\end{array}$ \\
\hline $\mathrm{PO}$ & $\begin{array}{l}\text { PO, TPP, } \\
\text { NTPP }\end{array}$ & $\begin{array}{l}\text { PO, TPP, } \\
\text { NTPP }\end{array}$ & $\begin{array}{l}\mathrm{DC}, \\
\mathrm{EC}, \mathrm{PO}, \\
\mathrm{TPP}^{-1} \\
\mathrm{NTPP}^{-1}\end{array}$ & $\begin{array}{l}\mathrm{DC}, \\
\mathrm{EC}, \mathrm{PO}, \\
\mathrm{TPP}^{-1}, \\
\text { NTPP }^{-1}\end{array}$ & $\mathrm{~T}$ & $\begin{array}{l}\mathrm{DC}, \\
\mathrm{EC}, \mathrm{PO}, \\
\mathrm{TPP}^{-1}, \\
\mathrm{NTPP}^{-1}\end{array}$ & $\begin{array}{l}\mathrm{DC}, \\
\mathrm{EC}, \mathrm{PO}, \\
\mathrm{TPP}^{-1}, \\
\mathrm{NTPP}^{-1}\end{array}$ \\
\hline $\mathrm{EC}$ & $\begin{array}{l}\text { PO, TPP, } \\
\text { NTPP }\end{array}$ & $\begin{array}{l}\text { EC, PO, } \\
\text { TPP, } \\
\text { NTPP }\end{array}$ & $\mathrm{DC}$ & $\mathrm{DC}, \mathrm{EC}$ & $\begin{array}{l}\mathrm{DC}, \mathrm{EC}, \\
\mathrm{PO}, \mathrm{TPP}, \\
\mathrm{NTPP}\end{array}$ & $\begin{array}{l}\mathrm{DC}, \mathrm{EC}, \\
\mathrm{PO}, \mathrm{TPP}, \\
\mathrm{TPP}^{-1} \\
\mathrm{EQ}\end{array}$ & $\begin{array}{l}\mathrm{DC}, \\
\mathrm{EC}, \mathrm{PO}, \\
\mathrm{TPP}^{-1}, \\
\mathrm{NTPP}^{-1}\end{array}$ \\
\hline $\mathrm{DC}$ & $\begin{array}{l}\text { DC, EC, } \\
\text { PO, TPP, } \\
\text { NTPP }\end{array}$ & $\begin{array}{l}\mathrm{DC}, \mathrm{EC}, \\
\mathrm{PO}, \mathrm{TPP}, \\
\text { NTPP }\end{array}$ & $\mathrm{DC}$ & $\mathrm{DC}$ & $\begin{array}{l}\text { DC, EC, } \\
\text { PO, TPP, } \\
\text { NTPP }\end{array}$ & $\begin{array}{l}\text { DC, EC, } \\
\text { PO, TPP, } \\
\text { NTPP }\end{array}$ & $\mathrm{T}$ \\
\hline
\end{tabular}

Table 6: The composition table of the eight base relations of the $R C C-8$ theory: the row gives the relation holding between $x$ and $y$; the column gives the relation holding between $y$ and $z$. The case contains the composition of $R_{1}(x, y)$ and $R_{2}(y, z)$, i.e. the set of the relations possibly holding between $x$ and $z$; the $\top$ mark corresponds to the undefined case, where all the relations of the $R C C$-8 theory may hold between $x$ et $z$. 


\section{Computing topological relations on raster images}

In order to classify spatial structures from maps or images, methods are needed to check spatial relations on the images. In this section, we propose computational operations on raster images: raster images are, in opposition to vector images, made of points, called pixels, characterized by their position (line, column) and their label (color or gray level). The computational operations are based on the interior and boundary sets of raster regions, leading to a fundamental problem of digital topology: defining the boundary of a raster region actually requires raster elements, that are two-dimensional and thus contradict the topology Kong and Rosenfeld 1989.

\subsection{The boundary problem}

When the raster representation is considered on its own, the boundary of a region can be defined by the pixels that are externally connected to the region: if the region is made of only one pixel, then its boundary is made of its eight neighbors. The boundary can also be internal, and composed of the pixels that are externally connected to the complementary set of the region (its exterior): then, if the region is a square made of nine pixels, its boundary contains eight pixels. These two definitions lead to topological paradoxes Kong and Rosenfeld 1989. Furthermore, they do not meet the Clarke's definition of connection, $x$ and $y$ share a point $:$ if the boundary is internal, two externally connected regions do not share a boundary point; conversely, if the boundary is external, two disconnected regions may share a boundary point.

Nevertheless, computational operations have been defined with such raster boundaries Egenhofer and Sharma 1993. The regions considered are regular raster regions that are 4-connected, non empty, and with no hole. Due to these restrictions, the boundary of a region respects the topology. Besides, operations based on the intersection of the internal and boundary sets allow to distinguish the relations $E C(x, y)$ and $D C(x, y)$. For instance, in the case of an external boundary, $\partial x \cap y^{\circ}$ and $x^{\circ} \cap \partial y$ are empty when the two regions are disconnected, and non-empty when the two regions are connected.

Another way is to complete the raster representation with one-dimensional boundaries. In Kovalevsky 1989, the raster representation is completed with edges and nodes representing the boundary of the pixels, to become a full topological model. The same representation is used in [Winter and Frank 1999, and the topological relations are defined with functions that apply to pixels, edges and nodes.

Our proposition is to solve the boundary problem by introducing an abstract boundary whose elements are abstract pixels. An abstract pixel stands across four real pixel, as shown in Figure 3 An abstract pixel $f$ is a boundary pixel of a region $x$ if there is at least one pixel belonging to $x$ and one pixel belonging to the complement of $x$ among the four real pixels intersected by $f$. The boundary intersection of two regions is then easily obtained, as shown in Figure 4 .

This representation is well adapted for the computation of topological relations since we deal with two raster representations. It can be applied either to 8-connected or to 


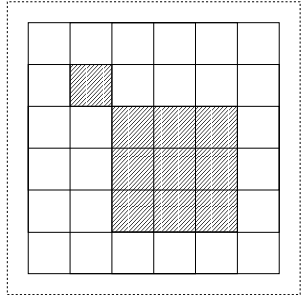

(a)

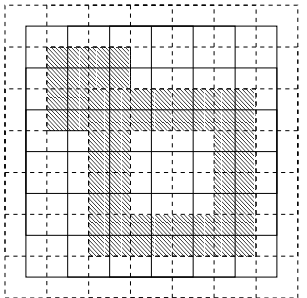

(b)

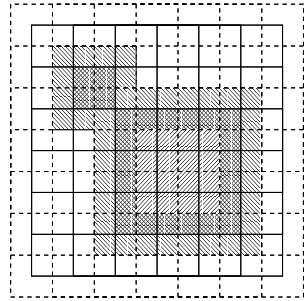

(c)

Figure 3: Defining the boundary of a region: (a) the interior of a region is made of real pixels (hatched); (b) the boundary of the region is made of abstract pixels standing across the real pixels (inverse hatched); (c) the combination of the interior and the boundary Mangelinck 1998.
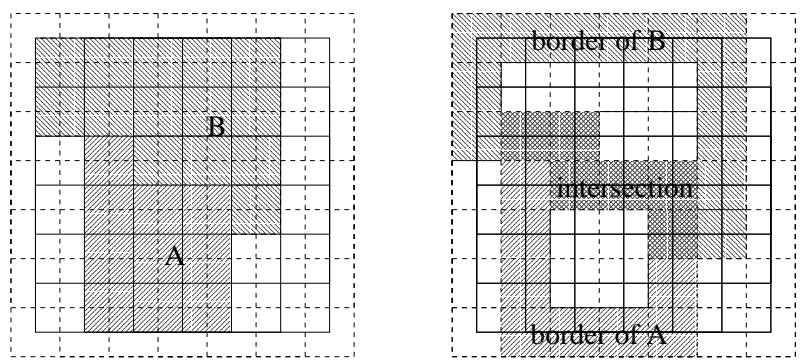

Figure 4: The boundary intersection of two regions on a raster image: the plain grid corresponds to the real pixels, the dotted grid corresponds to the abstract pixels.

4-connected regions. In both cases, the boundary is made of 4-connected abstract pixels. An abstract pixel of a boundary may have more than 2 neighbors (see figure 3), thus the boundary is not a Jordan curve as it is classically defined for digital topology [Latecki et al.1995. A possible solution is to eliminate some configurations, as it is done in Egenhofer and Sharma 1993, but this leads to eliminate 8-connected regions, which are frequent on land-use maps. Another solution is to define an oriented tracing of the boundary (e.g. first right and up), as it is proposed in Kovalevsky 1989. This problem should be analysed further, but this is not the purpose of the present paper. Actually, the fact that the boundary of a region is generally not a Jordan curve, is not a real problem for the intersection of boundaries, that is one of the basic operation used below. 


\subsection{Computational operations for defining topological relations}

In Egenhofer 1989, computational operations are defined for topological relations: the computational operations are the intersection of the interiors of two regions, $x^{\circ} \cap y^{\circ}$; the intersection of the boundaries of two regions, $\partial x \cap \partial y$; the intersections of the boundary of a region and the interior of the other region, $\partial x \cap y^{\circ}$ and $x^{\circ} \cap \partial y$. We call $C E-8$ the set of the eight conditions derived from these operations, according to their result (empty or not empty, see Table 5 .

These operations are unfortunately not convenient for our problem because of the definition of the region boundary. The interior and boundary sets are actually of different nature (real pixels versus abstract pixels) and it is thus incorrect to intersect those two sets. We propose a method for checking relations similar to the method of [Egenhofer 1989 but we use the set-difference of the interior sets rather than the intersections of boundary set and interior set. The regions of concern are closed, non empty and not necessarily internally connected. We propose to use the following four computational operations on these regions: the intersection of the interior sets, $x^{\circ} \cap y^{\circ}$; the intersection of the boundary sets, $\partial x \cap \partial y$; the two differences of the interior sets, $x^{\circ}-y^{\circ}$ and $y^{\circ}-x^{\circ}$. From these four operations we have derived the eight following conditions:

- $\left(x^{\circ}-y^{\circ}=\emptyset\right)$ : “ $x$ is a part of $y$ ”, denoted by $P(x, y) \sqrt{1}$

- $\left(x^{\circ}-y^{\circ} \neq \emptyset\right)$ : “ $x$ is not a part of $y$ ", denoted by $D x(x, y)$

- $\left(y^{\circ}-x^{\circ}=\emptyset\right)$ : “ $x$ contains $y$ " ( $y$ is a part of $x$ "), denoted by $P^{-1}(x, y)$

- $\left(y^{\circ}-x^{\circ} \neq \emptyset\right)$ : " $x$ does not contain $y$ " ( $y$ is not a part of $x$ "), denoted by $D y(x, y)$

- $\left(x^{\circ} \cap y^{\circ}=\emptyset\right)$ : " $x$ is discrete from $y$ ", denoted by $D R(x, y)$

- $\left(x^{\circ} \cap y^{\circ} \neq \emptyset\right)$ : “x overlaps $y$ ", denoted by $O(x, y)$

- $(\partial x \cap \partial y=\emptyset)$ : " $x$ does not share a boundary with $y$ ", denoted by $N A(x, y)$

- $(\partial x \cap \partial y \neq \emptyset)$ : “x shares a boundary with $y$ ”, denoted by $A(x, y)$

This set of conditions is called $C M-8$. It is important to notice that some conditions imply others because of the properties of the image regions. For instance, each of the conditions $x^{\circ}-y^{\circ}=\emptyset$ or $y^{\circ}-x^{\circ}=\emptyset$ implies the condition $x^{\circ} \cap y^{\circ} \neq \emptyset$ (the regions are non empty); the condition $\partial x \cap \partial y \neq \emptyset$ is implied by the conjunction of the same conditions (the regions are non empty and closed). Finally these four operations allow to check the $R C C-8$ theory relations on the images. The correspondence between each relation and the conditions are described on Table[7 each relation is equivalent to a subset of conditions of $C M$-8. Note that, if $P O(x, y)$ holds, $A(x, y)$ or $N A(x, y)$ may hold when $x$ or $y$ are not internally connected. However, since the $R C C$-8 theory does not distinguish different kind of partial overlaps, assuming $A(x, y)$ is not conflicting. Computing $D x(x, y), D y(x, y)$ and $O(x, y)$ is actually enough to check $P O(x, y)$.

Four conditions of $C M-8$ are identical to four conditions of $C E-8$ : those concerning the intersection of the interior sets and the intersection of the boundary sets of two regions. We

\footnotetext{
${ }^{1}$ The regions are closed and thus $x^{\circ}-y^{\circ}=\emptyset \leftrightarrow x-y=\emptyset$.
} 


\begin{tabular}{|l|c|c|c|c|}
\hline $\mathrm{x}, \mathrm{y}$ & $x^{\circ}-y^{\circ}$ & $y^{\circ}-x^{\circ}$ & $x^{\circ} \cap y^{\circ}$ & $\partial x \cap \partial y$ \\
\hline$E Q(x, y)$ & $\emptyset$ & $\emptyset$ & $\neq \emptyset$ & $\neq \emptyset$ \\
$\operatorname{NTPP}(x, y)$ & $\emptyset$ & $\neq \emptyset$ & $\neq \emptyset$ & $\emptyset$ \\
$T P P(x, y)$ & $\emptyset$ & $\neq \emptyset$ & $\neq \emptyset$ & $\neq \emptyset$ \\
$\operatorname{NTPP} P^{-1}(x, y)$ & $\neq \emptyset$ & $\emptyset$ & $\neq \emptyset$ & $\emptyset$ \\
$T P P^{-1}(x, y)$ & $\neq \emptyset$ & $\emptyset$ & $\neq \emptyset$ & $\neq \emptyset$ \\
$P O(x, y)$ & $\neq \emptyset$ & $\neq \emptyset$ & $\neq \emptyset$ & $\neq \emptyset$ \\
$E C(x, y)$ & $\neq \emptyset$ & $\neq \emptyset$ & $\emptyset$ & $\neq \emptyset$ \\
$D C(x, y)$ & $\neq \emptyset$ & $\neq \emptyset$ & $\emptyset$ & $\emptyset$ \\
\hline
\end{tabular}

Table 7: The correspondence between our computational operations for checking relations on the image and the $R C C-8$ theory relations Mangelinck 1998. See Table[5 for a comparison with the operations proposed in Egenhofer 1989.

have eliminated the conditions concerning the intersection of the boundary set of a region and the interior set of the other region. We have in turn added conditions concerning the set-difference of the interior sets of two regions. As previously said, this choice has been done in order to have consistent operations involving sets of the same kind : interiors (real pixels) or boundaries (abstract pixels).

It can be noticed that Ligozat has recently proposed to use the regions themselves and their interiors to compute the relations of the $R C C-8$ theory [Ligozat 1999. The operations he uses are the intersection and the inclusion. Thus, the relation $\operatorname{TPP}(x, y)$ is defined with $\left\{x \subset y, x \nsubseteq y^{\circ}\right\}$. This approach could also be used with raster images but the boundary problem should be reexamined.

\section{Two lattices of topological relations}

In this section, we introduce two lattices of topological relations that we describe according to their abilities for representing the relations, their reasoning properties and the associated computational operations. The first lattice is based on the power-set of the so-called set $\mathcal{B}$ of the eight base relations of the $R C C-8$ theory. The second lattice is a Galois lattice, that is based both on the set $\mathcal{B}$ and on the set $C M-8$ of conditions. In the following, we denote by $\mathcal{D}$ a set of spatial regions that are non empty, closed, and non necessarily internally connected. The definitions and proofs of this section are detailed in the appendix.

\subsection{A lattice based on the subsets of $\mathcal{B}$}

The first lattice that we have built is a "reference" lattice, denoted by $\mathcal{T}_{\mathcal{P}}$ and based on the power-set of $\mathcal{B}$. Its cardinality is $2^{8}=256$. The ordering relation is the set-inclusion. This lattice is defined as follows: 
Definition 1 The lattice $\mathcal{T}_{\mathcal{P}}$ is the structure $\langle R C C-8, \subseteq, \cap, \cup, \emptyset, \mathcal{B}\rangle$ where:

- the set of elements is the power-set of $\mathcal{B}$, denoted by $R C C-8$,

- the ordering is the set-inclusion $\subseteq$,

- the greatest lower bound (glb) of two elements $E_{1}$ and $E_{2}$ is the intersection $\cap$ of the sets $E_{1}$ and $E_{2}$,

- the least upper bound (lub) of two elements $E_{1}$ and $E_{2}$ is the union $\cup$ of the sets $E_{1}$ and $E_{2}$,

- the bottom element is the empty-set $\emptyset$,

- the top element is the set $\mathcal{B}$.

This lattice is actually associated to the boolean algebra $\left(R C C-8, \cap, \cup,{ }^{c}, \emptyset, \mathcal{B}\right)$ (see Davey and Priestley 1990). It is complemented: every element $E$ has a complement in $\mathcal{T}_{\mathcal{P}}$, which is its complement $E^{c}$ in $\mathcal{B}$. It contains all the subsets of $\mathcal{B}$ and thus represents all the compositions of $\mathcal{B}$ relations (see Table 6 ). In order to exploit this lattice we have defined a function $\pi_{r}$ that maps an element $E$ of $\mathcal{T}_{\mathcal{P}}$ to the disjunction of all the relations of $E$ (denoted by $\left.\pi_{r}(E)\right)$ :

$$
\forall(x, y) \in \mathcal{D}^{2}, \quad \pi_{r}(E)(x, y)={ }_{\text {def }} \bigvee_{r \in E} r(x, y)
$$

It can be noticed that the relation $\pi_{r}(E)$ associated to the $l u b E$ of two elements $E_{1}$, $E_{2}$, is the disjunction of the two relations $\pi_{r}\left(E_{1}\right), \pi_{r}\left(E_{2}\right)$ :

$$
\pi_{r}(E)=\pi_{r}\left(E_{1} \cup E_{2}\right)=\pi_{r}\left(E_{1}\right) \vee \pi_{r}\left(E_{2}\right)
$$

Furthermore, the ordering on the lattice elements is equivalent to the implication between relations. Firstly, the relation $\pi_{r}(E)$ associated to $E$ is implied by the relation $\pi_{r}\left(E^{\prime}\right)$ associated to a subset $E^{\prime}$ of $E$. Thus:

$$
\forall E, E^{\prime} \in \mathcal{T}_{\mathcal{P}}:\left(E^{\prime} \subseteq E\right) \rightarrow\left(\forall(x, y) \in \mathcal{D}^{2}, \pi_{r}\left(E^{\prime}\right)(x, y) \rightarrow \pi_{r}(E)(x, y)\right)
$$

Secondly the inverse implication is demonstrated thanks to the disjoint-property of the base relations of the $R C C-8$ theory: if a relation of $\mathcal{B}$ is true for a pair of regions of $\mathcal{D}^{2}$, the seven other relations are false. Then, if a disjunction of elements of $\mathcal{B}$ implies another disjunction, the elements of the first subset are necessarily elements of the second subset. Finally we prove the following theorem:

$$
\forall E, E^{\prime} \in \mathcal{T}_{\mathcal{P}}:\left(E^{\prime} \subseteq E\right) \leftrightarrow\left(\forall(x, y) \in \mathcal{D}^{2}, \pi_{r}\left(E^{\prime}\right)(x, y) \rightarrow \pi_{r}(E)(x, y)\right)
$$

For example, the element ( $T P P, N T P P)$ is included in the element ( $T P P, N T P P, E Q)$. The first one is associated to the relation $P P(x, y)$ while the second one is associated to the relation $P(x, y): P P(x, y) \rightarrow P(x, y)$ (see Table!). 


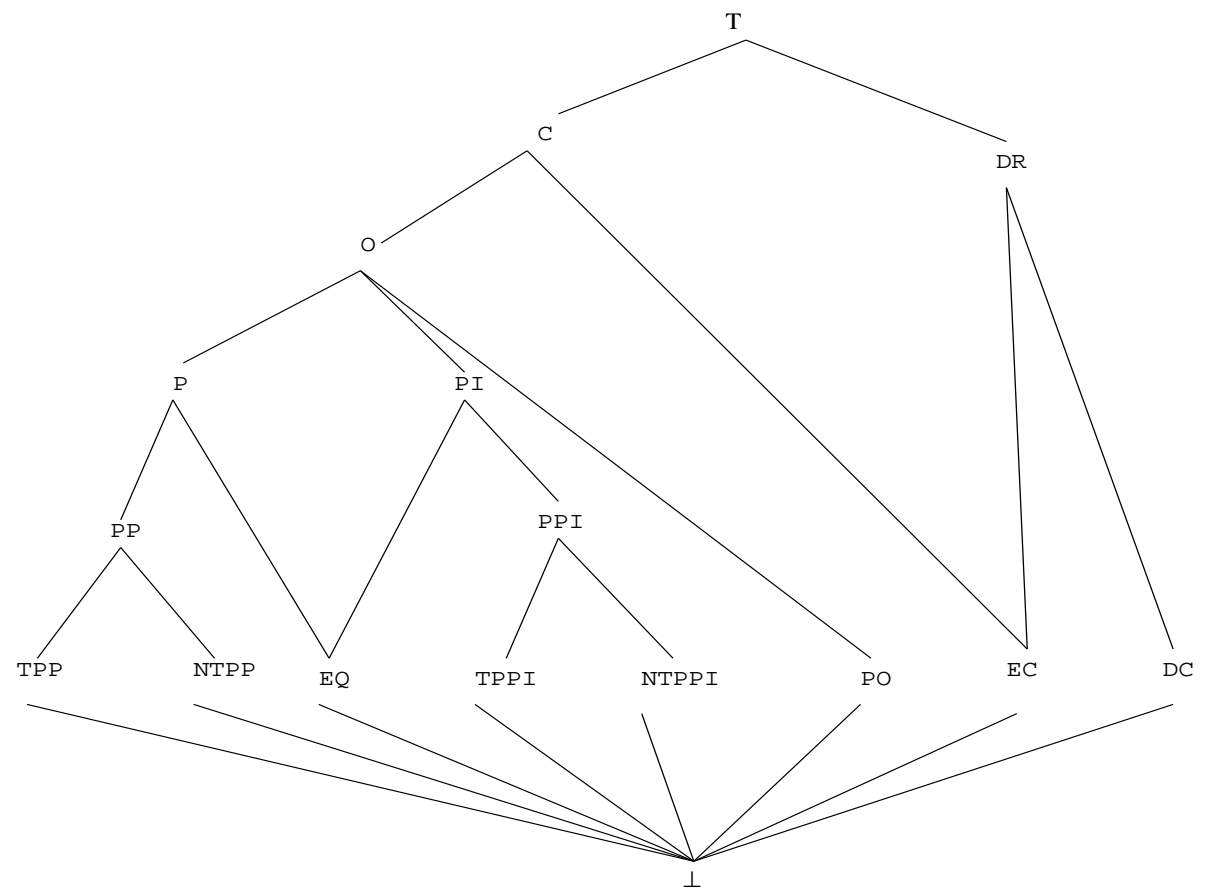

Figure 5: The lattice described in Randell and Cohn 1992. The labels denote relations that are disjunctions of $\mathcal{B}$ elements. This lattice is automatically completed into a boolean (i.e. distributive and complemented) lattice to be exploited.

A boolean lattice of topological relations similar to the lattice $\mathcal{T}_{\mathcal{P}}$ has been defined in [Randell and Cohn 1992] (see Figure[5. This lattice is used to prove theorems and to infer rules of composition between relations. The composition of all relations can actually be deduced from this lattice and from Table 6 For instance, if it is known that, for the three regions $x, y$ and $z, P P(x, y)$ and $E C(y, z)$, it can be inferred that $D R(x, z)(D R$ is the $l u b$ of the pair $(E C, D C)$, see Figure 5 :

$$
\begin{array}{rr}
\text { from the lattice: } & P P(x, y)=T P P(x, y) \vee N T T P(x, y) \\
\text { from the table: } & T P P(x, y) \circ E C(y, z)=D C(x, z) \vee E C(x, z) \\
\text { and: } & N T P P(x, y) \circ E C(y, z)=D C(x, z) \\
\text { thus: } & P P(x, y) \circ E C(y, z)=D C(x, z) \vee E C(x, z)=D R(x, z)
\end{array}
$$




\subsection{A smooth introduction to the Galois theory and formal concept analysis}

This introduction to the Galois lattice theory is based on Barbut and Monjardet 1970 , Guénoche and Van Mechelen 1993.

A number of classification problems can be formalized by means of a class of objects and a class of attributes, and a binary connection between the two classes, which indicates for each object-attribute pair whether the attribute applies to the object or not. The attributes may be features that are present or absent, or attribute values that have been dichotomized into boolean variables. From such a binary connection, it is possible to derive for each object $o$ its attribute pattern, i.e. the set of all attributes that apply to $o$, denoted by $A(o)$. Similarly, it is possible to derive for each attribute $a$ its object pattern, i.e. the set of all objects to which $a$ applies, denoted by $O(a)$. One may further derive rectangles, i.e. pairs $O \times A$ of an object set $O$ and an attribute set $A$, such that every attribute of $A$ applies to every object of $O$. Moreover, maximal rectangles $O \times A$ have the additional property that the attribute set $A$ consists of all common attributes of the objects of $O$, and that the object set $O$ consists of all objects to which the attributes of $A$ jointly apply.

Maximal rectangles are called formal concepts within the context of the binary connection under study [Davey and Priestley 1990, Ganter and Wille 1999]. They are called concepts because they actually represent a class of objects, the object set $O$ being the extension of the class, denoted by $\operatorname{Ext}(C)$ for a concept $C$, and the attribute set the intension of the class, denoted by $\operatorname{Int}(C)$. They are called formal concepts because they are mathematical entities that do not necessarily refer to a psychological reality. On the set of all concepts derived from a given binary connection, a partial order may be defined based on the set inclusion between the intensions (or between the extensions in a dual way). For this partial order, each pair of formal concepts has a least upper bound $(l u b)$, and a greatest lower bound $(g l b)$ : hence, formal concepts are organized within a lattice. More precisely, the partial order between two concepts $C_{1}$ and $C_{2}$, denoted by $C_{1} \sqsubseteq C_{2}$, verifies that the intension of $C_{2}$ (the subsumer concept) is included in the intension of $C_{1}$ (the subsumee concept), i.e. Int $\left(C_{2}\right) \subseteq \operatorname{Int}\left(C_{1}\right)$, or, dually, that the extension of $C_{1}$ is included in the extension of $C_{2}$, i.e. $\operatorname{Ext}\left(C_{1}\right) \subseteq \operatorname{Ext}\left(C_{2}\right)$. Thus, there exists an order-reversing one-to-one connection between the extensions and the intensions of formal concepts. This connection meets the criteria of a Galois connection (made precise below) and the lattice of formal concepts is called a Galois lattice Barbut and Monjardet 1970.

From a mathematical point of view, let $E$ and $F$ be two finite sets and $R$ a binary relation on $E \times F$. One may define a mapping $f: 2^{E} \longrightarrow 2^{F}$ such that, if $x$ is an element of $E$, $f(\{x\})$ consists of all elements of $F$ related to $x$ by $R$. Furthermore, if $X$ is an arbitrary part of $E, f(X)=\{y \in F \mid \forall x \in X: x R y\}$. Dually, we may define a mapping $g: 2^{F} \longrightarrow 2^{E}$ such that, if $y$ is an element of $F, g(\{y\})$ consists of all elements of $E$ that are related to $y$ by $R$. Furthermore, if $Y$ is an arbitrary part of $F, g(Y)=\{x \in E \mid \forall y \in Y: x R y\}$. The couple $\{f, g\}$ is said to be a Galois connection between the sets $E$ and $F$.

In terms of objects and attributes, $f(X)$ is the set of all attributes shared by all objects in $X$, and $g(Y)$ is the set of all objects that have all attributes of $Y$. Moreover, $X \subseteq X^{\prime} \Rightarrow$ 
$f\left(X^{\prime}\right) \subseteq f(X)$, and $Y \subseteq Y^{\prime} \Rightarrow g\left(Y^{\prime}\right) \subseteq g(Y)$ : the mappings $f$ and $g$ are decreasing. By applying first $f$ and then $g$, one may construct a composed mapping $h=g[f]=g \circ f$, which maps every part of $E$ onto a part of $E$. Similarly, by applying first $g$ and then $f$, one may construct a composed mapping $h^{\prime}=f[g]=f \circ g$, which maps every part of $F$ onto a part of $F$. It is then possible to show that $h$ (and respectively $h^{\prime}$ ) is: (i) monotonously increasing, that is if $X$ and $X^{\prime}$ are subsets of $E: X \subseteq X^{\prime} \Rightarrow h(X) \subseteq h\left(X^{\prime}\right)$, (ii) extensive, that is $X \subseteq h(X)$, and (iii) idempotent, that is $h(X)=h[h(X)]$. Furthermore, $h$ and $h^{\prime}$ are closure operators: a subset $X$ of $E$ is said to be closed if and only if $X=h(X) ; Y=h^{\prime}(Y)$ for a subset $Y$ of $F$. The closure operators $h$ for $E$ and $h^{\prime}$ for $F$ are said to be Galois closures.

Let $L_{E}$ and $L_{F}$ the sets of all closed parts of $E$ and $F$ (respectively), partially ordered by set inclusion. Equipped with this partial order, $L_{E}$ and $L_{F}$ have lattice structures: the $g l b$ of two parts is their intersection, whereas the $l u b$ of two parts is the closure of their union. The Galois connection $\{f, g\}$ restricted to the closed parts of $E$ and $F$ is a one-to-one correspondence between the lattices $L_{E}$ and $L_{F}$.

We may now consider the set $L$ of all couples of corresponding parts of $L_{E}$ and $L_{F}$, i.e. each element of $L$ is the Cartesian product of closed parts of $E$ and $F$, which may be denoted by $X \times f(X)$, or also, $g(Y) \times Y$, with $X, f(X), Y$, and $g(Y)$ being closed. We may further define the partial order relation $\sqsubseteq$ on $L: X \times Y \sqsubseteq X^{\prime} \times Y^{\prime}$ if and only if $X^{\prime} \subseteq X$ and $Y \subseteq Y^{\prime}$. The structure $(L, \sqsubseteq)$ is the Galois lattice of the relation $R$ on $E \times F$, and it can be demonstrated that the elements of $L$ are the formal concepts derived from the relation $R$.

\subsection{A Galois lattice based on topological relations}

\subsubsection{The design of the lattice}

Regarding our needs, we must build a lattice that respects the following requirements: it should contain the $\mathcal{B}$ and $C M-8$ sets, it should be ordered by the implication relation between the eight relations of $\mathcal{B}$ and the eight conditions of $C M-8$. Such a lattice can be built thanks to the Galois theory as described above. We have defined a Galois connection $\{f, g\}$ between $\mathcal{B}$ and $C M-8$ as follows:

Definition $2 f$ maps a relation $r$ of $\mathcal{B}$ to a subset of $C M-8$ where all conditions hold for a pair $(x, y)$ whenever the relation $r$ holds for $(x, y)$ :

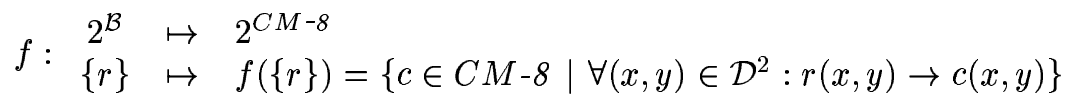

Furthermore, $f$ maps every subset $R$ of $\mathcal{B}$ to the subset $C$ of $C M-8$ whose conditions are implied by all the relations of $R: f(R)=\cap_{r \in R} f(\{r\})$.

Definition $3 \mathrm{~g}$ maps a condition $c$ of $C M-8$ to the subset of $\mathcal{B}$ where all relations imply the condition $c$ :

$$
\begin{aligned}
g: 2^{C M-8} & \mapsto 2^{\mathcal{B}}=R C C-8 \\
\{c\} & \mapsto g(\{c\})=\left\{r \in \mathcal{B} \mid \forall(x, y) \in \mathcal{D}^{2}: r(x, y) \rightarrow c(x, y)\right\}
\end{aligned}
$$

RR $n^{\circ} 4321$ 
Le Ber, Mangelinck \& Napoli

Furthermore $g$ maps every subset $C$ of $C M-8$ to a subset $R$ of $\mathcal{B}$ whose relations imply all the conditions of $C: g(C)=\cap_{c \in C} g(\{c\})$. The Galois connection $\{f, g\}$ is expressed in Table 8

\begin{tabular}{l|cccccccc} 
& $P$ & $D x$ & $P^{-1}$ & $D y$ & $D R$ & $O$ & $N A$ & $A$ \\
\hline$E Q$ & 1 & 0 & 1 & 0 & 0 & 1 & 0 & 1 \\
$N T P P$ & 1 & 0 & 0 & 1 & 0 & 1 & 1 & 0 \\
$T P P$ & 1 & 0 & 0 & 1 & 0 & 1 & 0 & 1 \\
$N T P P^{-1}$ & 0 & 1 & 1 & 0 & 0 & 1 & 1 & 0 \\
$T P P^{-1}$ & 0 & 1 & 1 & 0 & 0 & 1 & 0 & 1 \\
$P O$ & 0 & 1 & 0 & 1 & 0 & 1 & 0 & 1 \\
$E C$ & 0 & 1 & 0 & 1 & 1 & 0 & 0 & 1 \\
$D C$ & 0 & 1 & 0 & 1 & 1 & 0 & 1 & 0
\end{tabular}

Table 8: The table represents elements $(r, c)$, with $r \in \mathcal{B}$ (line) and $c \in C M-8$ (column). An element $(r, c)=1$ if $\forall(x, y) \in \mathcal{D}^{2}, r(x, y) \rightarrow c(x, y)$. Otherwise $(r, c)=0$.

The two functions $f$ and $g$ are used to define a closure operator $h$ of $R C C$ - 8 and a closure operator $h^{\prime}$ of $2^{C M-8}$ :

$$
h: \begin{aligned}
R C C-8 & \mapsto R C C-8 \\
h(R) & =g \circ f(R)
\end{aligned} \quad h^{\prime}: \begin{aligned}
2^{C M-8} & \mapsto 2^{C M-8} \\
h^{\prime}(C) & =f \circ g(C)
\end{aligned}
$$

The Galois lattice $\mathcal{T}_{\mathcal{G}}$ is built on the basis of the two closure operators $h$ and $h^{\prime}$. Its elements are pairs $(C, R) \in 2^{C M-8} \times R C C$ - 8 , where $h(R)=R$ and $C=f(R)=h^{\prime}(C)$. Such a pair is also a formal concept derived from the implication relation between $\mathcal{B}$ and $C M-8, C$ being the intension and $R$ the extension of this concept. $\mathcal{T}_{\mathcal{G}}$ is defined as follows:

Definition 4 The Galois lattice $\mathcal{T}_{\mathcal{G}}$ based on the implication between the relations of $\mathcal{B}$ and the conditions of $C M-8$ is the structure $\left\langle\mathcal{E}_{\mathcal{G}}, \sqsubseteq, \frown, \smile,(C M-8, \emptyset),(\emptyset, \mathcal{B})\right\rangle$ where:

- $\mathcal{E}_{\mathcal{G}}$ is the set of all pairs $(C, R)$ where $R$ is a subset of $\mathcal{B}$ closed for $h, C$ is a subset of $C M-8$ closed for $h^{\prime}, f(R)=C$ and $g(C)=R$.

- The ordering $\sqsubseteq$ between two elements $\left(C_{1}, R_{1}\right)$ and $\left(C_{2}, R_{2}\right)$ is defined as follows:

$$
\left(\left(C_{1}, R_{1}\right) \sqsubseteq\left(C_{2}, R_{2}\right)\right) \leftrightarrow\left\{\begin{array}{l}
C_{2} \subseteq C_{1} \\
R_{1} \subseteq R_{2}
\end{array}\right.
$$

where $\subseteq$ is the set-inclusion.

- The greatest lower bound (denoted by $\frown$ ) of two elements is defined as follows:

$$
\left(C_{1}, R_{1}\right) \frown\left(C_{2}, R_{2}\right)=\left(h^{\prime}\left(C_{1} \cup C_{2}\right), R_{1} \cap R_{2}\right)
$$




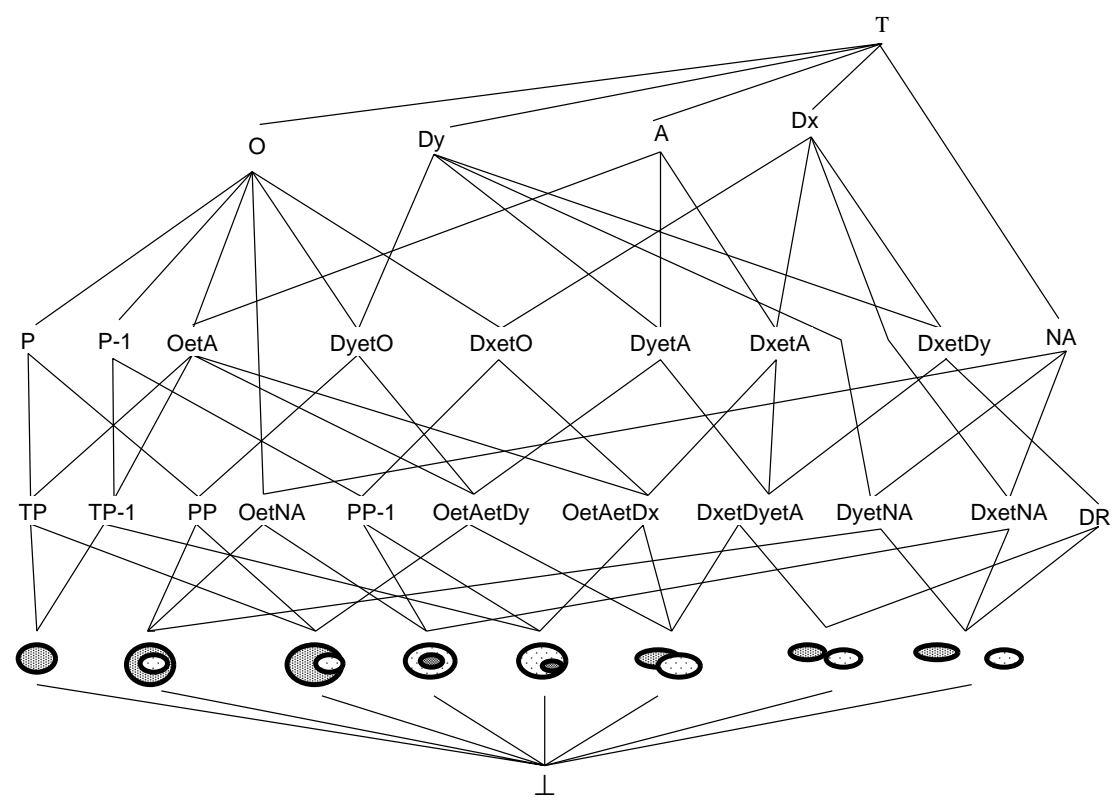

Figure 6: The Galois lattice built on the basis of Table 8 the pairs $(C, R)$ are denoted by the relation, or by the condition, to which they are associated by the relations $\pi_{r}$, or $\pi_{c}$ (see Section 3.3.2.

- The least upper bound (denoted by $\smile$ ) of two elements is defined as follows:

$$
\left(C_{1}, R_{1}\right) \smile\left(C_{2}, R_{2}\right)=\left(C_{1} \cap C_{2}, h\left(R_{1} \cup R_{2}\right)\right)
$$

where $\cup$ and $\cap$ are the set-union and the set-intersection.

- The bottom element is $(C M-8, \emptyset)$,

- The top element is $(\emptyset, \mathcal{B})$.

In a more concrete way, the Galois lattice $\mathcal{T}_{\mathcal{G}}$ is built by searching for the maximal rectangles in Table 8 or by searching for the subsets of $\mathcal{B}$ that are closed with respect to $h$. The lattice $\mathcal{T}_{\mathcal{G}}$ is shown in Figure [6] the denotations of the nodes are explained below. 


\subsubsection{The lattice ordering and the implication between relations}

As we have done before for the $\mathcal{T}_{\mathcal{P}}$ lattice (see paragraph 3.1) we now define the $\pi_{r}$ function that maps an element $(C, R)$ of $\mathcal{T}_{\mathcal{G}}$ to the disjunction of the relations of $R$ (denoted $\pi_{r}(C, R)$ ):

$$
\forall(x, y) \in \mathcal{D}^{2}, \quad \pi_{r}(C, R)(x, y)={ }_{\text {def }} \bigvee_{r \in R} r(x, y)
$$

Similarly we introduce the $\pi_{c}$ function that is concerned with the conditions of $C M-8$. The $\pi_{c}$ function maps an element $(C, R)$ of $\mathcal{T}_{\mathcal{G}}$ to the conjunction of the $C$ elements:

$$
\forall(x, y) \in \mathcal{D}^{2}, \quad \pi_{c}(C, R)(x, y)={ }_{d e f} \bigwedge_{c \in C} c(x, y)
$$

We show below that the two formulas resulting from the application of $\pi_{c}$ and $\pi_{r}$ are equivalent. This equivalence is used to name the elements of $\mathcal{T}_{\mathcal{G}}$, either as a disjunction of relations or as a conjunction of conditions. On Figure 6 elements are denoted by a name or an icon: a name represents a conjunction of conditions (e.g. OetA denotes the element $(\{O, A\}, R)$, where et stands for "and") or a disjunction of relations (e.g. PP denotes the element $(C,\{T P P, N T P P\})$, since $P P=T P P \vee N T T P) 2$. The icons at the bottom of the lattice represent the elements associated to the eight base relations of the $R C C-8$ theory (e.g. $\mathrm{EQ}=\left(\left\{O, A, P, P^{-1}\right\},\{E Q\}\right)$.

The $\pi_{c}$ and $\pi_{r}$ functions are also used to make explicit the link between the lattice ordering and the implication relation on the topological relations. Actually the following property is proved for all pairs of elements of $\mathcal{T}_{\mathcal{G}}$ :

$$
\left(C_{1}, R_{1}\right) \sqsubseteq\left(C_{2}, R_{2}\right) \leftrightarrow\left\{\begin{array}{l}
\forall(x, y) \in \mathcal{D}^{2}, \bigvee_{r \in R_{1}} r(x, y) \rightarrow \bigvee_{r \in R_{2}} r(x, y) \\
\forall(x, y) \in \mathcal{D}^{2}, \bigwedge_{c \in C_{1}} c(x, y) \rightarrow \bigwedge_{c \in C_{2}} c(x, y)
\end{array}\right.
$$

Thus, if an element $\mathrm{E}_{1}=\left(C_{1}, R_{1}\right)$ (e.g. EQ) is smaller than another element $\mathrm{E}_{2}=\left(C_{2}, R_{2}\right)$ (e.g. TP), the disjunction of the relations of $R_{1}$ implies the disjunction of the relations of $R_{2}$ and the conjunction of the conditions of $C_{1}$ implies the conjunction of the conditions of $C_{2}$ :

$$
\begin{aligned}
& \mathrm{EQ} \sqsubseteq \mathrm{TP} \leftrightarrow \quad \forall(x, y)(E Q(x, y) \rightarrow(E Q \vee T P P)(x, y)) \\
& \text { and } \quad\left(\left(A \wedge P \wedge P^{-1} \wedge O\right)(x, y) \rightarrow(A \wedge P \wedge O)(x, y)\right)
\end{aligned}
$$

\subsection{Properties of the $\mathcal{T}_{\mathcal{G}}$ lattice}

\subsubsection{The characteristics of the elements of $\mathcal{T}_{\mathcal{G}}$}

The following properties characterize the elements of $\mathcal{T}_{\mathcal{G}}$ :

\footnotetext{
${ }^{2}$ In the following $A$ denotes the condition and $\mathrm{A}$ denotes the lattice element where the condition is represented (respectively EC and EC to denote the topological relation and the element of the lattice).
} 
Property 1 If a pair $\mathrm{E}=(C, R)$ is an element of the $\mathcal{T}_{\mathcal{G}}$ lattice, then the relation $\pi_{r}(\mathrm{E})=$ $\pi_{r}(C, R)$ is equivalent to the condition $\pi_{c}(\mathrm{E})=\pi_{c}(C, R)$ :

$$
(C, R) \in \mathcal{E}_{\mathcal{G}} \rightarrow\left(\forall(x, y) \in \mathcal{D}^{2}, \bigvee_{r \in R} r(x, y) \leftrightarrow \bigwedge_{c \in C} c(x, y)\right)
$$

This property shows that the relation $\pi_{r}(\mathrm{E})$ associated with an element of $\mathcal{T}_{\mathcal{G}}$ is logically equivalent to the condition $\pi_{c}(\mathrm{E})$ associated with the same element. Thus, checking a relation on the image requires to look for the element of $\mathcal{T}_{\mathcal{G}}$ that represents this relation and to check the associated conditions.

The second property that characterizes the elements of the $\mathcal{T}_{\mathcal{G}}$ lattice is as follows:

Property 2 Let $R$ be a subset of $\mathcal{B}$ and $C$ a subset of $C M-8$. If the relation formed by the disjunction of the elements of $R$ is equivalent to the condition formed by the conjunction of the elements of $C$, then the pair $(\bar{C}, R)$, where $\bar{C}=h^{\prime}(C)$, is an element of $\mathcal{T}_{\mathcal{G}}$ :

$$
\left(\forall(x, y) \in \mathcal{D}^{2}, \bigvee_{r \in R} r(x, y) \leftrightarrow \bigwedge_{c \in C} c(x, y)\right) \rightarrow(\bar{C}, R) \in \mathcal{T}_{\mathcal{G}}
$$

The next property characterizes the elements of $\mathcal{B}$. It can be deduced from Property 2 or by examining Table 8

Property 3 For each element $r$ of $\mathcal{B},(f(\{r\}),\{r\})$ is an element of $\mathcal{T}_{\mathcal{G}}$.

Property 3 shows that the base relations of $\mathcal{B}$ are represented as individuals in the $\mathcal{T}_{\mathcal{G}}$ lattice, situated at the bottom of the lattice, as displayed in Figure 6. The next property characterizes the conditions of $C M-8$ and their representation in $\mathcal{T}_{\mathcal{G}}$ :

Property 4 Each condition $c$ of $C M-8$ can be associated to a distinct element of $\mathcal{T}_{\mathcal{G}}, \mathrm{E}_{\mathrm{c}}=$ $(\bar{C}, R)$, where $R=g(\{c\}), \bar{C}=h^{\prime}(\{c\})$, and:

$$
\forall(x, y) \in \mathcal{D}^{2}, c(x, y) \leftrightarrow \bigvee_{r \in R} r(x, y) \leftrightarrow \bigwedge_{c_{i} \in \bar{C}} c_{i}(x, y)
$$

This last property can directly be checked on the conditions. In particular:

$$
\begin{array}{lll}
g(\{P\})=\{E Q, N T P P, T P P\} & & \\
h^{\prime}(\{P\})=(P, O) & \text { and } \quad P(x, y) \leftrightarrow P(x, y) \wedge O(x, y) \\
g\left(\left\{P^{-1}\right\}\right)=\left\{E Q, N T P P^{-1}, T P P^{-1}\right\} & & \\
h^{\prime}\left(\left\{P^{-1}\right\}\right)=\left(P^{-1}, O\right) & \text { and } \quad P^{-1}(x, y) \leftrightarrow P^{-1}(x, y) \wedge O(x, y) \\
g(\{D R\})=\{D C, E C\} & & \\
h^{\prime}(\{D R\})=(D R, D x, D y) & \text { and } \quad D R(x, y) \leftrightarrow D R(x, y) \wedge D x(x, y) \wedge D y(x, y)
\end{array}
$$

The other singletons of $2^{C M-8}$ are closed for $h^{\prime}: h^{\prime}(\{c\})=\{c\}$. Thus, all conditions of $C M-8$ are represented as individuals in the $\mathcal{T}_{\mathcal{G}}$ lattice. They are represented respectively by the elements P, $\mathrm{P}^{-1}, \mathrm{DR}, \mathrm{O}, \mathrm{Dy}, \mathrm{A}, \mathrm{Dx}$ and NA (see Figure[6]. 
Le Ber, Mangelinck \& Napoli

\subsubsection{Characterizing the $g l b(\frown)$}

The operator $\frown$ in the lattice is equivalent to the operator $\wedge$ (conjunction) on the relations.

Property 5 An element $\mathrm{E}$ of $\mathcal{T}_{\mathcal{G}}$ is the glb of two elements $\mathrm{E}_{1}$ and $\mathrm{E}_{2}$ if and only if $\pi_{r}(\mathrm{E})$ is equivalent to the conjunction of the two relations $\pi_{r}\left(\mathrm{E}_{1}\right)$ and $\pi_{r}\left(\mathrm{E}_{2}\right)$ :

$$
\left(\mathrm{E}=\mathrm{E}_{1} \frown \mathrm{E}_{2}\right) \leftrightarrow\left(\forall(x, y) \in \mathcal{D}^{2}, \pi_{r}(\mathrm{E})(x, y) \leftrightarrow \pi_{r}\left(\mathrm{E}_{1}\right)(x, y) \wedge \pi_{r}\left(\mathrm{E}_{2}\right)(x, y)\right)
$$

Furthermore, each element $\mathrm{E}$ of $\mathcal{T}_{\mathcal{G}}$ is characterized by the equivalence between the disjunction $\pi_{r}(\mathrm{E})$ and the conjunction $\pi_{c}(\mathrm{E})$. Thus:

Property 6 Let $\mathrm{E}, \mathrm{E}_{1}, \mathrm{E}_{2}$ be three elements of the $\mathcal{T}_{\mathcal{G}}$ lattice:

$$
\left(\mathrm{E}=\mathrm{E}_{1} \frown \mathrm{E}_{2}\right) \leftrightarrow\left(\forall(x, y) \in \mathcal{D}^{2}, \pi_{c}(\mathrm{E})(x, y) \leftrightarrow \pi_{c}\left(\mathrm{E}_{1}\right)(x, y) \wedge \pi_{c}\left(\mathrm{E}_{2}\right)(x, y)\right)
$$

\subsubsection{The minimal set of conditions for checking a relation}

The relations expressed in the $\mathcal{T}_{\mathcal{G}}$ lattice are associated to a set $C$ of conditions which are sufficient to check this relation. But all conditions of $C$ are not necessary, and some of these conditions should be eliminated to minimize the calculation time. Let us take an example: $E Q(x, y)$, " $x$ is identical with $y$ ", is associated to the set of conditions $C=\left\{A, O, P, P^{-1}\right\}$ (see Table 8 ). But, the checking of $E Q(x, y)$ only relies on the checking of the conditions $P(x, y)$ (" $x$ is a part of $y$ ") and $P^{-1}(x, y)$ (" $x$ contains $y$ ") since the conjunction of these two conditions imply the two other conditions $A(x, y)$ (" $x$ shares a boundary with $y$ ") and $O(x, y)$ (" $x$ overlaps $y$ "). This implication is due to the properties of the image regions (non-emptiness, closure) as previously mentioned. In $\mathcal{T}_{\mathcal{G}}, E Q$ is represented by the element $\mathrm{EQ}=\left(\left\{A, O, P, P^{-1}\right\},\{E Q\}\right)$. According to Property 1

$$
\forall(x, y) \in \mathcal{D}^{2}, E Q(x, y) \leftrightarrow\left(A \wedge O \wedge P \wedge P^{-1}\right)(x, y)
$$

Furthermore, $P$ and $P^{-1}$ are represented by the elements $\mathrm{P}$ and $\mathrm{P}^{-1}$. The $g l b$ of $\mathrm{P}$ and $\mathrm{P}^{-1}$ is EQ (see Figure 6 ). According to Property 6

$$
\forall(x, y) \in \mathcal{D}^{2}, \pi_{c}(\mathrm{EQ})(x, y) \leftrightarrow \pi_{c}(\mathrm{P})(x, y) \wedge \pi_{c}\left(\mathrm{P}^{-1}\right)(x, y)
$$

Then (Property 4$): \forall(x, y) \in \mathcal{D}^{2},\left(A \wedge O \wedge P \wedge P^{-1}\right)(x, y) \leftrightarrow\left(P \wedge P^{-1}\right)(x, y)$

Then: $\forall(x, y) \in \mathcal{D}^{2}, E Q(x, y) \leftrightarrow\left(P \wedge P^{-1}\right)(x, y)$

Finally, the properties of the operator $\frown$ can be used to find out the minimal set of conditions that are necessary to check the $E Q$ relation. This minimal set can directly be read on the lattice. 
The minimal sets associated to the other relations of $\mathcal{B}$ can be found accordingly:

$$
\begin{aligned}
\forall(x, y) \in \mathcal{D}^{2}: \quad N T P P(x, y) & \leftrightarrow & (P \wedge N A)(x, y) \\
T P P(x, y) & \leftrightarrow & (P \wedge A \wedge D y)(x, y) \\
N T P P^{-1}(x, y) & \leftrightarrow & \left(P^{-1} \wedge N A\right)(x, y) \\
T P P^{-1}(x, y) & \leftrightarrow & \left(P^{-1} \wedge A \wedge D x\right)(x, y) \\
P O(x, y) & \leftrightarrow & (D x \wedge D y \wedge O)(x, y) \\
E C(x, y) & \leftrightarrow & (A \wedge D R)(x, y) \\
D C(x, y) & \leftrightarrow & (N A \wedge D R)(x, y)
\end{aligned}
$$

\subsubsection{Characterizing the $l u b(\smile)$}

Property 7 For all pairs of lattice elements $\left(\mathrm{E}_{1}, \mathrm{E}_{2}\right)$, the lub $\mathrm{E}^{\prime}=\mathrm{E}_{1} \smile \mathrm{E}_{2}$ is such that the disjunction of $\pi_{r}\left(\mathrm{E}_{1}\right)$ and $\pi_{r}\left(\mathrm{E}_{2}\right)$ implies the relation $\pi_{r}\left(\mathrm{E}^{\prime}\right)$ :

$$
\left(\mathrm{E}^{\prime}=\mathrm{E}_{1} \smile \mathrm{E}_{2}\right) \rightarrow\left(\forall(x, y) \in \mathcal{D}^{2}, \pi_{r}\left(\mathrm{E}^{\prime}\right)(\mathrm{x}, \mathrm{y}) \leftarrow \pi_{\mathrm{r}}\left(\mathrm{E}_{1}\right)(\mathrm{x}, \mathrm{y}) \vee \pi_{\mathrm{r}}\left(\mathrm{E}_{2}\right)(\mathrm{x}, \mathrm{y})\right)
$$

This property is easy to prove since the relation set $R^{\prime}$ of the element $\mathrm{E}^{\prime}=\mathrm{E}_{1} \smile \mathrm{E}_{2}$ is the closure of the union of the two relation sets $R_{1}, R_{2}$ of the elements $\mathrm{E}_{1}$ and $\mathrm{E}_{2}$ (cf. Definition 4). The reciprocal property of Property $\mathbf{Z}$ is not true in the $\mathcal{T}_{\mathcal{G}}$ lattice because an element of $\mathcal{T}_{\mathcal{G}}$ can be the $l u b$ of several elements: $\mathrm{E}^{\prime}$ can be both the $l u b$ of the pair $\mathrm{E}_{1}, \mathrm{E}_{2}$ and the $l u b$ of the pair $\mathrm{E}_{3}, \mathrm{E}_{4}$. Then:

$$
\begin{gathered}
\mathrm{E}^{\prime}=\mathrm{E}_{1} \smile \mathrm{E}_{2}: \pi_{r}\left(\mathrm{E}_{1}\right) \vee \pi_{\mathbf{r}}\left(\mathrm{E}_{2}\right) \rightarrow \pi_{\mathbf{r}}\left(\mathrm{E}^{\prime}\right) \\
\mathrm{E}^{\prime}=\mathrm{E}_{3} \smile \mathrm{E}_{4}: \pi_{r}\left(\mathrm{E}_{2}\right) \vee \pi_{\mathrm{r}}\left(\mathrm{E}_{4}\right) \rightarrow \pi_{\mathbf{r}}\left(\mathrm{E}^{\prime}\right) \\
\text { and } \pi_{r}\left(\mathrm{E}^{\prime}\right) \rightarrow \pi_{\mathbf{r}}\left(\mathrm{E}_{1}\right) \vee \pi_{\mathbf{r}}\left(\mathrm{E}_{2}\right) \vee \pi_{\mathbf{r}}\left(\mathrm{E}_{3}\right) \vee \ldots
\end{gathered}
$$

For instance, the $l u b$ of the elements $\mathrm{TP}$ and $\mathrm{TP}^{-1}$ of $\mathcal{T}_{\mathcal{G}}$ is the element 0etA which is also the $l u b$ of the elements DetAetDx and DetAetDy (see Figure 6). This property has to be linked to a property of closed sets: the lub of two closed sets is generally not a closed set, whereas the $g l b$ is a closed set [Barbut and Monjardet 1970, Davey and Priestley 1990.

\section{Elements for the comparison of the lattices}

\subsection{Comparing the lattices $\mathcal{T}_{\mathcal{P}}$ and $\mathcal{T}_{\mathcal{G}}$}

From an implementation point of view, the $\mathcal{T}_{\mathcal{G}}$ lattice is more easy to manage than the $\mathcal{T}_{\mathcal{P}}$ lattice. An object-based knowledge representation system must be reasonably sized in terms of memory space. The $\mathcal{T}_{\mathcal{P}}$ lattice is complemented and contains $2^{8}=256$ elements. This means that the designer has to implement and to manage 256 classes of relations. The $\mathcal{T}_{\mathcal{G}}$ lattice by contrast contains only 34 elements (see Figure6). Thus, it can be worth to choose the $\mathcal{T}_{\mathcal{G}}$ lattice rather than the $\mathcal{T}_{\mathcal{P}}$ lattice.

Another major advantage of the $\mathcal{T}_{\mathcal{G}}$ lattice is the representation of the conditions that are used to check the relations on images, and the representation of the implication relations 
Le Ber, Mangelinck \& Napoli

holding between the conditions and the relations. This is not done in the $\mathcal{T}_{\mathcal{P}}$ lattice: the conditions are only implicitly represented, as they are equivalent to disjunctions of $\mathcal{B}$ relations. The implication relations between the topological relations and the conditions thus do not appear in the $\mathcal{T}_{\mathcal{P}}$ lattice, making this lattice not very useful for checking relations on the image. On the contrary, the links between relations and conditions are singled out in the $\mathcal{T}_{\mathcal{G}}$ lattice, which is built from the Galois connection between $\mathcal{B}$ and $C M-8$.

The last point is about reasoning in the lattices: the reasoning is complete in $\mathcal{T}_{\mathcal{P}}$ whereas it is not in $\mathcal{T}_{\mathcal{G}}$. One of the reasons is that some compositions of the base relations are missing in $\mathcal{T}_{\mathcal{G}}$. In this case, the $\mathcal{T}_{\mathcal{P}}$ lattice has to be preferred: it includes all the disjunctions of $\mathcal{B}$ elements and thus contains all the compositions of Table 6. Conversely the $\mathcal{T}_{\mathcal{G}}$ lattice contains the following compositions (see Figure6):

$$
\begin{aligned}
P O \vee T P P \vee N T P P \vee T P P^{-1} \vee N T P P^{-1} \vee E Q & =\pi_{r}(\mathrm{0}) \\
D C \vee E C \vee P O \vee T P P \vee N T P P & =\pi_{r}(\mathrm{Dy}) \\
D C \vee E C \vee P O \vee T P P^{-1} \vee N T P P^{-1} & =\pi_{r}(\mathrm{Dx}) \\
P O \vee T P P \vee N T P P & =\pi_{r}(\text { Dyet0 }) \\
P O \vee T P P^{-1} \vee N T P P^{-1} & =\pi_{r}(\text { Dxet0 }) \\
P O \vee T P P \vee T P P^{-1} \vee E Q & =\pi_{r}(\text { DetA }) \\
N T P P \vee T P P & =\pi_{r}(\mathrm{PP}) \\
N T P P^{-1} \vee T P P^{-1} & =\pi_{r}\left(\mathrm{PP} \mathrm{P}^{-1}\right) \\
E C \vee D C & =\pi_{r}(\mathrm{DR})
\end{aligned}
$$

and lacks three compositions:

$$
\begin{array}{r}
E C \vee P O \vee N T P P \vee T P P \\
E C \vee P O \vee N T P P^{-1} \vee T P P^{-1} \\
D C \vee E C \vee P O \vee T P P \vee T P P^{-1} \vee E Q
\end{array}
$$

In order to combine the advantages of $\mathcal{T}_{\mathcal{G}}$ (computation) and the advantages of $\mathcal{T}_{\mathcal{P}}$ (inference), we propose to extend the $\mathcal{T}_{\mathcal{G}}$ lattice with the lacking compositions. The extended lattice is described in the next paragraph and is compared to the $\mathcal{T}_{\mathcal{G}}$ lattice.

\subsection{Extending the lattice $\mathcal{T}_{\mathcal{G}}$}

\subsubsection{The design of the extended lattice $\mathcal{T}_{\mathcal{G E}}$}

The extended lattice $\mathcal{T}_{\mathcal{G E}}$ is built progressively: the lacking relations are added step by step as it is done in incremental algorithms for building lattices [Missikoff and Scholl 1989. 
Carpineto and Romano 1993, Godin et al. 1995. The addition of a relation in $\mathcal{T}_{\mathcal{G E}}$ relies on two steps:

- Addition of the relation according to the order of the original lattice, i.e. searching for the most specific ascendants and the most general descendants of the new relation;

- Addition of a $g l b$ or of a $l u b$ for the pairs lacking one.

According to the first step, the addition of the two first relations relies on the following implications with the elements of the lattice $\mathcal{T}_{\mathcal{G}}$ :

$$
\begin{aligned}
E C \vee P O \vee N T P P \vee T P P & \rightarrow \pi_{r}(\text { Dy }) \\
E C \vee P O \vee N T P P \vee T P P & \leftarrow \pi_{r}(\text { Dyet0) } \\
E C \vee P O \vee N T P P \vee T P P & \leftarrow \pi_{r}(\text { DyetA }) \\
E C \vee P O \vee N T P P^{-1} \vee T P P^{-1} & \rightarrow \pi_{r}(\text { Dx }) \\
E C \vee P O \vee N T P P^{-1} \vee T P P^{-1} & \leftarrow \pi_{r}(\text { Dxet0) } \\
E C \vee P O \vee N T P P^{-1} \vee T P P^{-1} & \leftarrow \pi_{r}(\text { DxetA })
\end{aligned}
$$

Following these implications, the relation $E C \vee P O \vee N T P P \vee T P P$ is expressed by the element Dyet-Aou0- that is placed between the element Dy (up) and the elements DyetA and Dyet0 (down, see Figure 8 ). The relation $E C \vee P O \vee N T P P^{-1} \vee T P P^{-1}$, as for it, is expressed by the element Dxet-Aou0- that is placed between the element Dx (up) and the elements DxetA and Dxet0 (down). The lattice structure is then preserved and no other element has to be added.

The insertion of the last relation in the extended lattice is more complicated; according to the first step, we find the following implications with the elements of the lattice $\mathcal{T}_{\mathcal{G}}$ :

$$
\begin{aligned}
& D C \vee E C \vee P O \vee T P P \vee T P P^{-1} \vee E Q \rightarrow \pi_{r}(\mathrm{~A}) \\
& D C \vee E C \vee P O \vee T P P \vee T P P^{-1} \vee E Q \\
& D C \vee E C \vee P O \vee T P P \vee T P P^{-1} \vee E Q \rightarrow \top
\end{aligned}
$$

The relation is expressed by the element Aou-DxetDy- and placed in the lattice above the two elements A and DxetDy. The lattice structure is lost (see Figure[7, left):

- the pair (DyetA, DxetDy) has two lubs, Aou-DxetDy- and Dy,

- the pair (DxetA, DxetDy) has also two lubs, Aou-DxetDy- and Dx.

In order to preserve the structure of the lattice $\mathcal{T}_{\mathcal{G E}}$, two new elements have to be added, -AouDx-etDy, being the new lub of (DyetA, DxetDy), and -AouDy-etDx, being the new lub of (DxetA, DxetDy) (see Figure(7). Finally we have added five elements to $\mathcal{T}_{\mathcal{G}}$ to build $\mathcal{T}_{\mathcal{G E}}$. These five elements corresponds to the five following subsets of $\mathcal{B}$ :

1. Dyet-Aou0-: $(E C, P O, N T P P, T P P)$ 

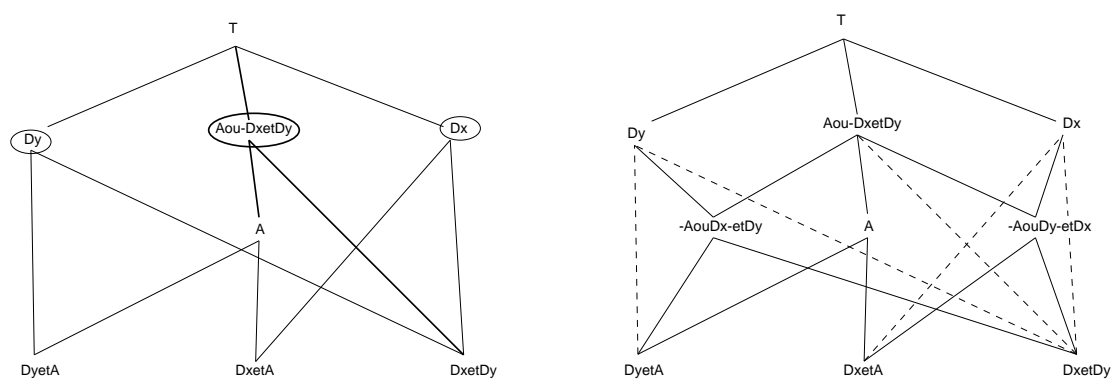

Figure 7: Left: adding the element Aou-DxetDy- in the lattice $\mathcal{T}_{\mathcal{G}}$ (partial view). The two lubs of the pairs (DyetA, DxetDy) and (DxetA, DxetDy) are encircled. Right: adding the two elements -AouDx-etDy and -AouDy-etDx to preserve the structure of the lattice.

2. Dxet-Aou0-: $\left(E C, P O, N T P P^{-1}, T P P^{-1}\right)$

3. Aou-DxetDy-: $\left(D C, E C, P O, T P P, T P P^{-1}, E Q\right)$

4. -AouDy-etDx: $\left(D C, E C, P O, T P P^{-1}\right)$

5. -AouDx-etDy: $(D C, E C, P O, T P P)$

No other element has to be added and the lattice structure is preserved. The lattice $\mathcal{T}_{\mathcal{G E}}$ is displayed in Figure8

Finally, the lattice $\mathcal{T}_{\mathcal{G E}}$ contains all the necessary relations for representing the compositions of $\mathcal{B}$ relations. The lattice $\mathcal{T}_{\mathcal{G E}}$ is thus sufficient to infer the compositions of all disjunctions of $\mathcal{B}$ relations from the distributivity of o with respect to $\vee$ (cf. Section 3.1 ).

\subsubsection{Comparing $\mathcal{T}_{\mathcal{G}}$ and $\mathcal{T}_{\mathcal{G E}}$}

Since we want to represent the relations with computation and inference capabilities, we can work either with $\mathcal{T}_{\mathcal{G}}$ or $\mathcal{T}_{\mathcal{G E}}$. The last one seems preferable since it contains all the relations for representing the compositions of $\mathcal{B}$ relations: thus all possible relations can be inferred from what is already computed on the image. Furthermore, the $\mathcal{T}_{\mathcal{G E}}$ lattice contains a reasonable number of relations and can be easily managed in an object-based knowledge representation system.

Besides, one should notice that the ordering in $\mathcal{T}_{\mathcal{G E}}$ is distinct from the one in $\mathcal{T}_{\mathcal{G}}$ that is defined by the inclusion relation both on $C M-8$ subsets and on $\mathcal{B}$ subsets (cf. Definition 4 ). The lattice $\mathcal{T}_{\mathcal{G E}}$ is an extension of $\mathcal{T}_{\mathcal{G}}$ and is actually based on the implication between relations, being equivalent to the inclusion on $\mathcal{B}$ subsets, as it has been shown before (Section 3.3.2. But it is not possible to associate subsets of $C M-8$ to the five relations added to $\mathcal{T}_{\mathcal{G}}$ (to obtain $\mathcal{T}_{\mathcal{G E}}$ ) in the same way as it is done for the original elements of $\mathcal{T}_{\mathcal{G}}$. Actually the new elements of $\mathcal{T}_{\mathcal{G E}}$ are not formal concepts as introduced in Section 3.2 Thus the ordering of $\mathcal{T}_{\mathcal{G}}$ is not preserved in $\mathcal{T}_{\mathcal{G} \mathcal{E}}$. 


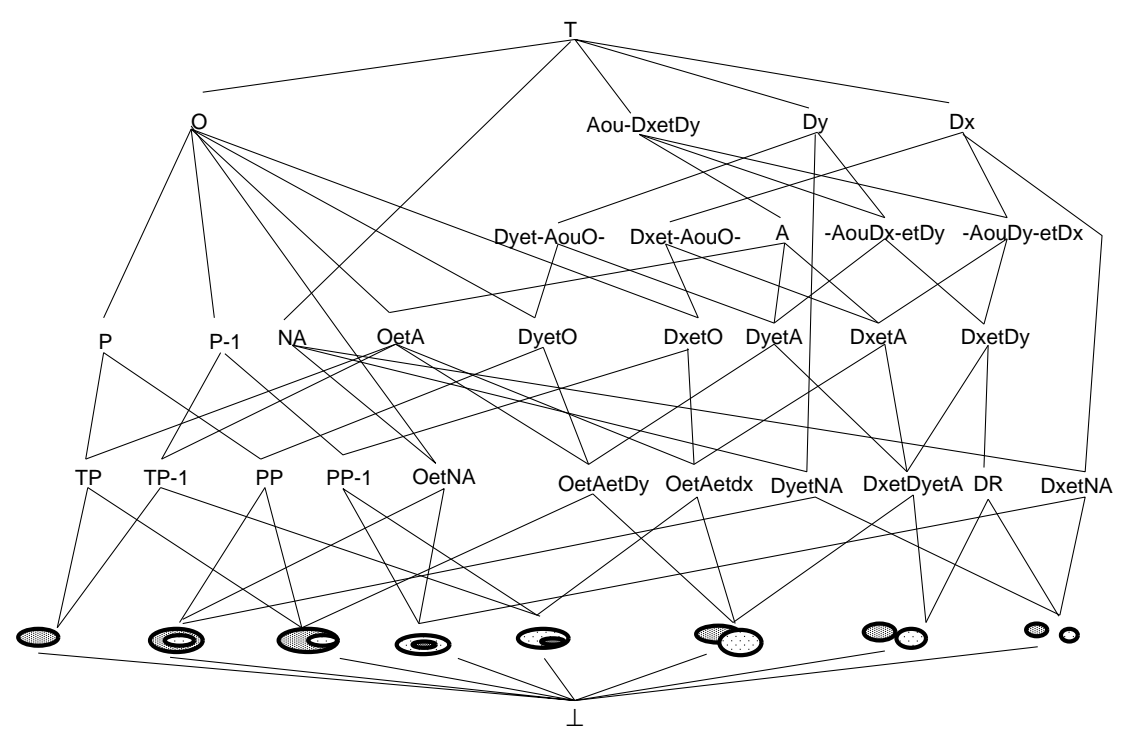

Figure 8: The lattice $\mathcal{T}_{\mathcal{G E}}$, extending $\mathcal{T}_{\mathcal{G}}$, for representing the compositions of $\mathcal{B}$ relations.

Therefore, the $\mathcal{T}_{\mathcal{G E}}$ lattice cannot be easily used for the computation of relations: the five added relations are not equivalent to a conjunction of conditions, and they cannot be associated to a minimal set of conditions. For example, the element Dxet-Aou0- is placed between the element DxetA and the element $D x$ in $\mathcal{T}_{\mathcal{G}}$ (see Figure 6 and 8 ). According to the $\mathcal{T}_{\mathcal{G}}$ ordering, the condition set $C$ of Dxet-Aou0- would be such as: $\{D x, A\} \subsetneq C \subsetneq\{D x\}$, and that is impossible.

\subsection{A second Galois lattice}

In this section we describe a second Galois lattice that is based on the operations proposed in Egenhofer 1989 to compute topological relations. In our present work, we have chosen to use other operations and we have built a Galois lattice to exploit the links between these operations and the $\mathcal{B}$ relations. It is interesting to build a Galois lattice based on another set of operations and to compare this new lattice to the Galois lattice $\mathcal{T}_{\mathcal{G}}$ in order to make explicit the strengths and the limitations of the two approaches from this point of view.

\subsubsection{The design of the lattice}

The eight conditions based on the operations proposed in Egenhofer 1989 have been described previously (see Section 2.2. Their set is named $C E-8$. Four of these conditions are identical to these of $C M-8(A, N A, D R, O)$. The four others are: 
$\partial x \cap y^{\circ}=\emptyset \quad$ denoted $N F O$

$\partial x \cap y^{\circ} \neq \emptyset \quad$ denoted $F O$

$x^{\circ} \cap \partial y=\emptyset \quad$ denoted NOF

$x^{\circ} \cap \partial y \neq \emptyset \quad$ denoted $O F$

The implication relation between these eight conditions and the relations of $\mathcal{B}$ has been made explicit in Table 5 A Galois connection $\{f, g\}$ can then be defined between the sets $C E-8$ and $\mathcal{B}$ as it is done in Section 3.3.1 The connection is described in Table 9 ,

\begin{tabular}{l|cccccccc} 
& $D R$ & $O$ & $N A$ & $A$ & $N F O$ & $F O$ & $N O F$ & $O F$ \\
\hline$D C$ & 1 & 0 & 1 & 0 & 1 & 0 & 1 & 0 \\
$E C$ & 1 & 0 & 0 & 1 & 1 & 0 & 1 & 0 \\
$P O$ & 0 & 1 & 0 & 1 & 0 & 1 & 0 & 1 \\
$T P P I$ & 0 & 1 & 0 & 1 & 1 & 0 & 0 & 1 \\
$T P P$ & 0 & 1 & 0 & 1 & 0 & 1 & 1 & 0 \\
$N T P P$ & 0 & 1 & 1 & 0 & 0 & 1 & 1 & 0 \\
$N T P P I$ & 0 & 1 & 1 & 0 & 1 & 0 & 0 & 1 \\
$E Q$ & 0 & 1 & 0 & 1 & 1 & 0 & 1 & 0 \\
\hline
\end{tabular}

Table 9: The table represents elements $(r, c)$, with $r \in \mathcal{B}$ (line) and $c \in C E-8$ (column). An element $(r, c)=1$ if $\forall(x, y) \in \mathcal{D}^{2}, r(x, y) \rightarrow c(x, y)$. Otherwise $(r, c)=0$.

The Galois lattice built from this connection is named $\mathcal{T}_{\mathcal{G}}$, its elements are pairs $(C, R)$, where $C$ is a subset of $C E-8$ and $R$ is a subset of $\mathcal{B}$. It is displayed in Figure 9 The notation of the elements is similar to the notation used for the $\mathcal{T}_{\mathcal{G}}$ lattice: an element is denoted with a name or a icon describing a relation, or with a name or a conjunction ("et" standing for "and") of names of conditions.

\subsubsection{Comparing the Galois lattices $\mathcal{T}_{\mathcal{G}}$ and $\mathcal{T}_{\mathcal{G}}$ '}

As they are both Galois lattices, the $\mathcal{T}_{\mathcal{G}}$ and $\mathcal{T}_{\mathcal{G}}$ ' lattices have basically the same properties. The main differences between the two lattices are about the conditions and the sets of relations that are represented.

Let us first examine the conditions of $C E-8$. Five of them are expressed within elements that are immediate descendants of $T$, the top element of the lattice (see Figure9). These elements are A, 0, NA, NFO, NOF, whose set of conditions $C=\{c\}$ is closed for $h^{\prime}$ :

$$
\forall c \in\{A, O, N A, N O F, N F O\}, h^{\prime}(\{c\})=\{c\}
$$

The other conditions behave as follows:

$$
\begin{aligned}
& h^{\prime}(\{O F\})=\{O F, O\} \quad \text { and } O F(x, y) \leftrightarrow O F(x, y) \wedge O(x, y) \\
& h^{\prime}(\{F O\})=\{F O, O\} \quad \text { and } F O(x, y) \leftrightarrow F O(x, y) \wedge O(x, y) \\
& h^{\prime}(\{D R\})=\{D R, N O F, N F O\} \quad \text { and } \quad D R(x, y) \leftrightarrow D R(x, y) \wedge N O F(x, y) \wedge N F O(x, y)
\end{aligned}
$$




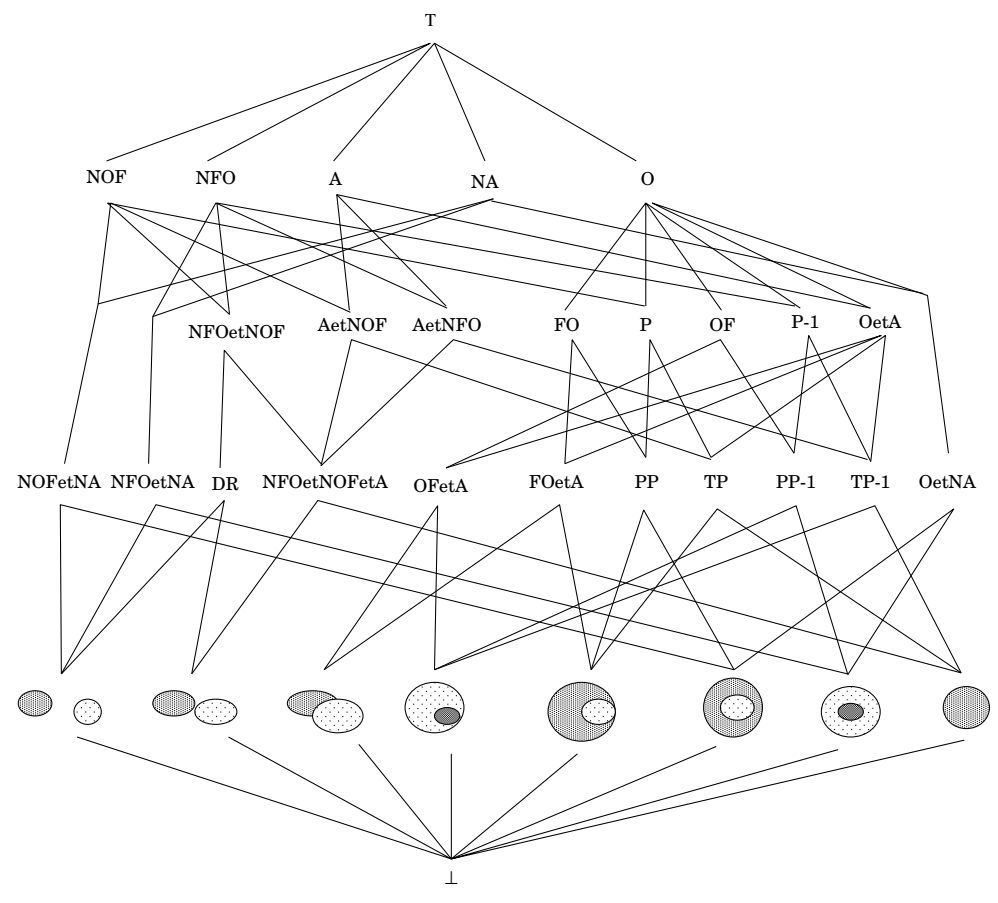

Figure 9: The Galois lattice $\mathcal{T}_{\mathcal{G}}$ ' relying on the conditions proposed in Egenhofer 1989.

We now compare the number of conditions used to check the relations in $\mathcal{T}_{\mathcal{G}}$ and $\mathcal{T}_{\mathcal{G}}$. Table 10 shows the number of conditions used for computing the base relations in each lattice. The first column gives the names of the corresponding elements in the two lattices ( $\mathrm{E}^{\prime}$ and E). The two following columns give the sets of conditions $C^{\prime}$ and $C$. The two last columns give the number $\left(n^{\prime}, n\right)$ of conditions used to check the relations (i.e. the cardinality of the minimal set of conditions, see Section 3.4.3). The number of conditions used to check all the relations expressed in the two lattices is described in the appendix.

Checking a relation does not necessarily require the same number of conditions in the two lattices (see Table 10): for instance, checking the relation $E Q$ relies on two conditions in $\mathcal{T}_{\mathcal{G}},\left\{P, P^{-1}\right\}$, whereas it needs three conditions in $\mathcal{T}_{\mathcal{G}}{ }^{\prime},\{O, N F O, N O F\}$. The conditions $P$ and $P^{-1}$ respectively correspond to the pairs $\{O, N F O\}$ and $\{O, N O F\}$. Conversely, checking the relation $P O$ relies on three conditions in $\mathcal{T}_{\mathcal{G}},\{D x, D y, A\}$, whereas it needs only the two conditions $\{F O, O F\}$ in $\mathcal{T}_{\mathcal{G}}$ '.

To summarize, the two lattices use the same mean number of conditions to check the relations, common or not common. Thus the choice between the two Galois lattices is not a matter of computation optimization (minimization of the number of tests) but rather a 


\begin{tabular}{|c|c|c|c|c|}
\hline $\mathrm{E}^{\prime} / \mathrm{E}$ & $C^{\prime}$ & $C$ & $n^{\prime}$ & $n$ \\
\hline $\mathrm{DC}$ & $\{D R, N O F, N F O, N A\}$ & $\{D R, D x, D y, N A\}$ & 2 & 2 \\
\hline EC & $\{D R, N O F, N F O, A\}$ & $\{D R, D x, D y, A\}$ & 2 & 2 \\
\hline PO & $\{F O, O F, O, A\}$ & $\{D x, D y, O, A\}$ & 2 & 3 \\
\hline $\mathrm{TPP}^{-1}$ & $\{O F, O, A, N F O\}$ & $\left\{P^{-1}, O, A, D x\right\}$ & 3 & 3 \\
\hline TPP & $\{F O, O, A, N O F\}$ & $\{P, O, A, D y\}$ & 3 & 3 \\
\hline NTPP & $\{F O, O, N A, N O F\}$ & $\{P, O, N A, D y\}$ & 2 & 2 \\
\hline $\mathrm{NTPP}^{-1}$ & $\{O F, O, N A, N F O\}$ & $\left\{P^{-1}, O, N A, D x\right\}$ & 2 & 2 \\
\hline EQ & $\{N F O, N O F, O, A\}$ & $\left\{P, P^{-1}, O, A\right\}$ & 3 & 2 \\
\hline
\end{tabular}

Table 10: Comparing the number of conditions used to check the base relations in the Galois lattices $\mathcal{T}_{\mathcal{G}}$ ' and $\mathcal{T}_{\mathcal{G}}:$ E' / E are the names of the elements in the two lattices, $n^{\prime}$ and $n$ the number of conditions used to check a relation (see the appendix for the complete comparison).

matter of adequation with the needs of the current application data: in one hand, it can be preferable to compute the intersection of a boundary set and an interior set $\left(\partial x \cap y^{\circ}\right.$, $x^{\circ} \cap \partial y$ ), in the other hand, it can be preferable to compute the difference of two interior sets $\left(x^{\circ}-y^{\circ}, y^{\circ}-x^{\circ}\right)$. According to our definition of the boundary of a region (Section 2.1), the second solution is better in our case.

\subsection{Reasoning with lattices of topological relations}

An important reasoning problem in the $R C C-8$ theory, denoted by RSAT, is deciding consistency of a set of constraints of the form $x R y$, where $R$ is a disjunction of the base relations, and $x, y$ are spatial regions. This problem is NP-hard in general, but there exist subsets of $R C C$ - 8 where it is tractable Renz and Nebel 1999, Renz 1999. Three maximal tractable subsets of $R C C-8$ containing all base relations are defined in [Renz 1999]. According to these results, we conclude that RSAT $\left(\mathcal{T}_{\mathcal{G}}\right)$ and $\operatorname{RSAT}\left(\mathcal{T}_{\mathcal{G}}{ }^{\prime}\right)$ are NP-hard since the two lattices contain the relations $\left\{N T P P, N T P P^{-1}\right\}$ (element DetNA) and $\left\{D C, N T P P, N T P P^{-1}\right\}$ (NA, see Appendix). All other relations represented in $\mathcal{T}_{\mathcal{G}}$ and $\mathcal{T}_{\mathcal{G}}$ ' are member of the maximal tractable subset $\widehat{\mathcal{H}}_{8}$ defined in [Renz and Nebel 1999, Renz 1999].

The constraint satisfaction problem has been examined for internally connected planar regions in Grigni et al.1995. The authors consider two notions of satisfiability, the relational consistency and the realizability. The realizability subproblem corresponds to planar constraints that must be satisfied besides the relational consistency ones, and it is shown to be almost everywhere NP-hard. According to the authors the realizability subproblem only appears for configurations involving more than 12 regions. Furthermore, in the case of regions that are not necessarily internally connected, relational consistency implies realizability [Renz 1998]. 
Lattices have been used for automatic theorem proving and relations infering in the framework of the $R C C-8$ theory [Randell and Cohn 1992]. Moreover, they are well adapted for classification-based reasoning. The Galois lattices in particular are well adapted for the classification and computation of topological relations. Their main drawback is that reasoning is not complete, even in the extended lattices like $\mathcal{T}_{\mathcal{G E}}$, due to the building method of these lattices and their resulting properties. Actually, the lattices are not complemented and the $l u b$ of two elements is not equivalent to the disjunction of the corresponding relations (see Section 3.4.4). For instance, if the relation $\left(T P \vee T P^{-1}\right)(x, y)$ holds, it is concluded in $\mathcal{T}_{\mathcal{G}}$ that $\left(T P P \vee T P P^{-1} \vee E Q \vee P O\right)(x, y)$ holds (DetA is the $l u b$ of $\mathrm{TP}$ and $\mathrm{TP}^{-1}$ ), whereas it is concluded in $\mathcal{T}_{\mathcal{P}}$ that $\left(T P P \vee T P P^{-1} \vee E Q\right)(x, y)$ holds. The last conclusion cannot be found in $\mathcal{T}_{\mathcal{G}}$ since the corresponding element does not exist.

This drawback is in balance with the low number of elements in the Galois lattices, making them practically more manageable than the boolean lattice $\mathcal{T}_{\mathcal{P}}$.

\section{Lattice-based classification of spatial relations and struc- tures}

The lattices $\mathcal{T}_{\mathcal{G}}$ and $\mathcal{T}_{\mathcal{G E}}$ have been implemented within the frame-based representation system Y3 [Ducournau 1991, Masini et al.1991. The elements of the lattices have been represented within relation classes organized according to the lattice ordering. In the following, the same notation is used for an element and the class representing this element, i.e. $\mathrm{E}$ stands for the element $\mathrm{E}$ and for the class representing $\mathrm{E}$.

\subsection{Inferences on the relations}

Figure 10 shows the generic class SPATIAL-RELATION that represents the top element of the lattice, and three other classes. The class 0 is a subclass of the generic class, the class DR is a subclass of the class DxetDy and the class EC is a subclass of the classes DR and DxetDyetA.

The classes are described with attributes representing the various properties of the lattice elements, such as the attributes complement, converse and condition. The value of the complement attribute of class $\mathrm{E}$ is the class $\mathrm{E}^{\mathrm{c}}$ such that $\pi_{r}\left(\mathrm{E}^{\mathrm{c}}\right)$ is the complement of the relation $\pi_{r}(\mathrm{E})$. For example the complement of the class 0 is the class DR since the two classes represent respectively the lattice elements $\left(\{O\},\left\{E Q, P O, N T P P, T P P, N T P P^{-1}\right.\right.$,$\left.\left.T P P^{-1}\right\}\right)$ and $(\{D R\},\{D C, E C\})$. The value of the converse attribute of a class $E$ is the element $\mathrm{E}^{-1}$ such that $\pi_{r}\left(\mathrm{E}^{-1}\right)$ is the converse relation of $\pi_{r}(\mathrm{E})$ (see Section 1.3). For example, the class EC fixes the value of the attribute converse: the converse relation of $\mathrm{EC}$ is EC itself. The value of the condition attribute of a class $\mathrm{E}$ is the set of conditions associated to this class. For example, the class 0 assigns the value of this attribute to $\mathrm{CO}$ that represents the condition $O(x, y)=\left(x^{\circ} \cap y^{\circ} \neq \emptyset\right)$.

The composition of relations is represented within a method named transitivity. This method is only defined in the classes that represent the $\mathcal{B}$ relations. The method is detailed 


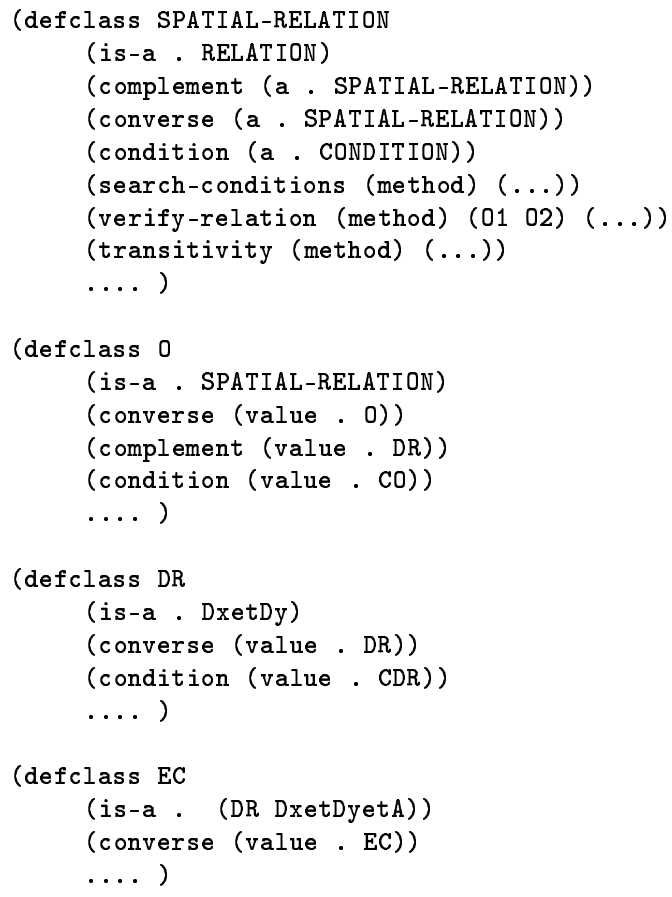

Figure 10: Classes representing the elements of the lattices $\mathcal{T}_{\mathcal{G}}$ or $\mathcal{T}_{\mathcal{G} \mathcal{E}}$. The properties of the relations are represented within attributes and methods.

for the class DC in Figure 11 for example the relation associated to DC, composed with the relation associated to EC gives the relation associated to Dy.

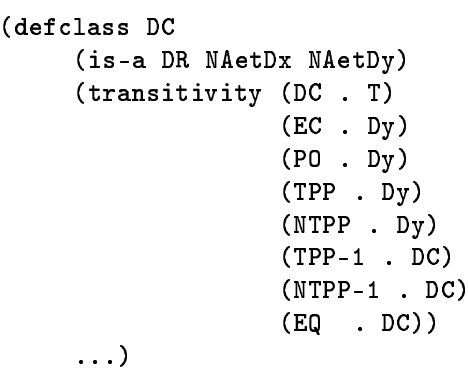

Figure 11: Representing the rules of composition within methods. T corresponds to the lattice element $T$. 
The computation of the composition of two arbitrary relations is done as follows: to each element $E_{i}$ of $\mathcal{T}_{\mathcal{G}}$ corresponds a disjunction of $\mathcal{B}$ relations $\left(\pi_{r}\left(E_{\mathbf{i}}\right)\right)$. Thus, a set of descendants $\mathcal{I}_{E_{i}}$ where the method transitivity is defined can be associated to each class $E_{i}$. For example, the class $D R$ is associated with the set $\mathcal{I}_{\mathrm{DR}}=\{\mathrm{DC}, \mathrm{EC}\}$. Considering the sets of descendants $\mathcal{I}_{E_{i}}$ for a class $E_{i}$ and $\mathcal{I}_{E_{j}}$ for a class $E_{j}$, it is possible to apply the transitivity method of all the classes of $\mathcal{I}_{E_{i}}$ to all the classes of $\mathcal{I}_{\mathrm{E}_{j}}$ and thus obtain a new set of classes. The lub of these classes is the class that represents the relation resulting from the composition of $\pi_{r}\left(\mathrm{E}_{\mathbf{i}}\right)$ and $\pi_{r}\left(\mathrm{E}_{\mathbf{j}}\right)$.

For example, the sets associated to DC and PP are $\mathcal{I}_{\mathrm{DC}}=\{\mathrm{DC}\}$ and $\mathcal{I}_{\mathrm{PP}}=\{\mathrm{TPP}, \mathrm{NTPP}\}$. Composing DC and PP, the system will apply the transitivity method of the class DC to the classes TPP and NTPP. The resulting set is $\{$ Dy, Dy $\}$ whose $l u b$ is Dy (see Figure 11).

\subsection{The computation of the relations}

As shown in Figure 10, each relation class $\mathrm{E}$ also includes an attribute condition recording the set of conditions of the lattice element $\mathrm{E}$. The value of this attribute is inherited from the classes Dx, Dy, 0, NA, A, (see Figure 6) where its value is fixed. The inheritance mechanism is cumulative for all classes, but $\mathrm{P}, \mathrm{P}^{-1}$ and $\mathrm{DR}$. In those last three classes, the value of condition is overridden with a unique condition: for example this value for DR is equal to CDR that corresponds to the condition $x^{\circ} \cap y^{\circ}=\emptyset$ (Figure 10). Finally, the classes where the value of condition is a singleton correspond to the singletons of $2^{C M-8}$.

We have previously shown that the conjunction $(\wedge)$ and the glb $(\frown)$ were equivalent in the $\mathcal{T}_{\mathcal{G}}$ lattice (Property [5). This property can be used to find out the minimal set of necessary conditions to check a relation $\pi_{r}(\mathrm{E})$, and, as well, to find out the classes representing this set among the ascendants of $\mathrm{E}$.

For example, let us suppose that the $E Q$ relation, expressed by the class EQ, has to be checked for two regions in the image; this class has a set of superclasses whose condition attribute value is reduced to a unique condition. This set is $\mathcal{S}=\left\{\mathrm{P}, \mathrm{P}^{-1}, \mathrm{~A}, 0\right\}$. The associated conditions are: $P, P^{-1}, A$ and $O$. The common descendants of the elements of $\mathcal{S}$ are then searched in the class hierarchy: $\mathrm{P}$ and $\mathrm{P}^{-1}$ have a common descendant (their $g l b$, in fact) that is EQ. Using Property 5 we deduce that the conjunction of the two conditions $P$ and $P^{-1}$ is equivalent to the relation $E Q$ (cf. Section 3.4.3). Thus, checking the relation $E Q$ can be reduced to checking the two conditions $P$ and $P^{-1}$.

Practically, the verify-relation methods of the classes $\mathrm{P}$ and $\mathrm{P}^{-1}$ are used on the image regions. These methods respectively compute the conditions $P$, or $P^{-1}$, associated to the classes; if they succeed, an instance of the $g l b$ of $\mathrm{P}$ and $\mathrm{P}^{-1}$ is created, i.e. an instance of $\mathrm{EQ}$ is created. If the verify-relation method of one of the classes fails then the complement attribute of this class can be used to find out which relation has been verified. For example, if the verify-relation method of $P$ fails, the system can infer that the relation Dx holds (the value of the complement attribute of $\mathrm{P}$ is $\mathrm{Dx}$, representing the condition $D x$ ); the system finally creates an instance of the $g l b$ of the two classes: $\mathrm{P}^{-1} \frown \mathrm{Dx}=\mathrm{PP}^{-1}$ (see Figure 6). 
Le Ber, Mangelinck \& Napoli

\subsection{Classification of spatial structures}

Let us return to our original motivation, i.e. to recognize spatial structures on land-use maps and to classify them according to landscape models. These models are represented within classes, and organized into several lattice structures. Model classes are linked through instances of the relation classes. For example (Figure 12), the class TERRITORY is specialized into the classes TERRITORY-DC-FOREST and TERRITORY-EC-FOREST, that are defined by the following attributes: $t-d c n-f$ and $t-e c n-f$, that are respectively instance of the class DC and instance of the class EC Mangelinck 1998.

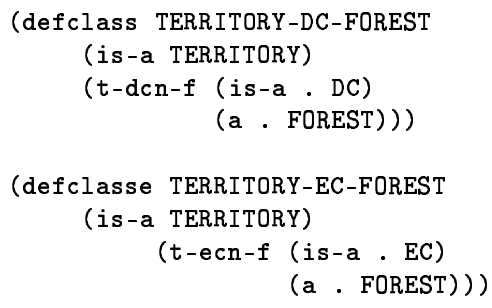

Figure 12: Examples of classes representing models of spatial structures. The attributes represent the relations between spatial entities: for instance the attribute $t-d c n-f$ is an instance of the class DC; its range is the class FOREST.

We have defined 6 terminal models that describe the main global structures of village territories in the Lorraine region (East of France) Le Ber and Mangelinck 1998. These models are built from more basic and general models (see Figure 13). For instance, the model VALLEY is a specification of the following models:

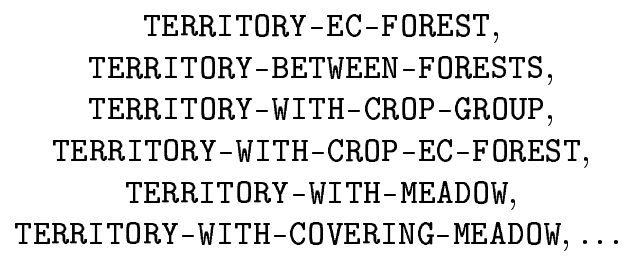

These models are linked to the models CROP, CROP-GROUP, CROP-EC-FOREST, MEADOW, COVERING-MEADOW, ... that are elements of the lattice describing the models of crop fields and meadows. This lattice itself contains about 140 elements.

The classification of a spatial structure requires the classification of a set of related spatial structures and the corresponding relations, as it is illustrated in the following simplified example. The system first recognizes a territory entity on the image thanks to its label (the image that must be analyzed has been previously labelled [Bachacou 1995]). The system represents this entity as an instance, say t1, of the model class TERRITORY. The system tries then to classify the instance t1 into a more specific class, for example the 


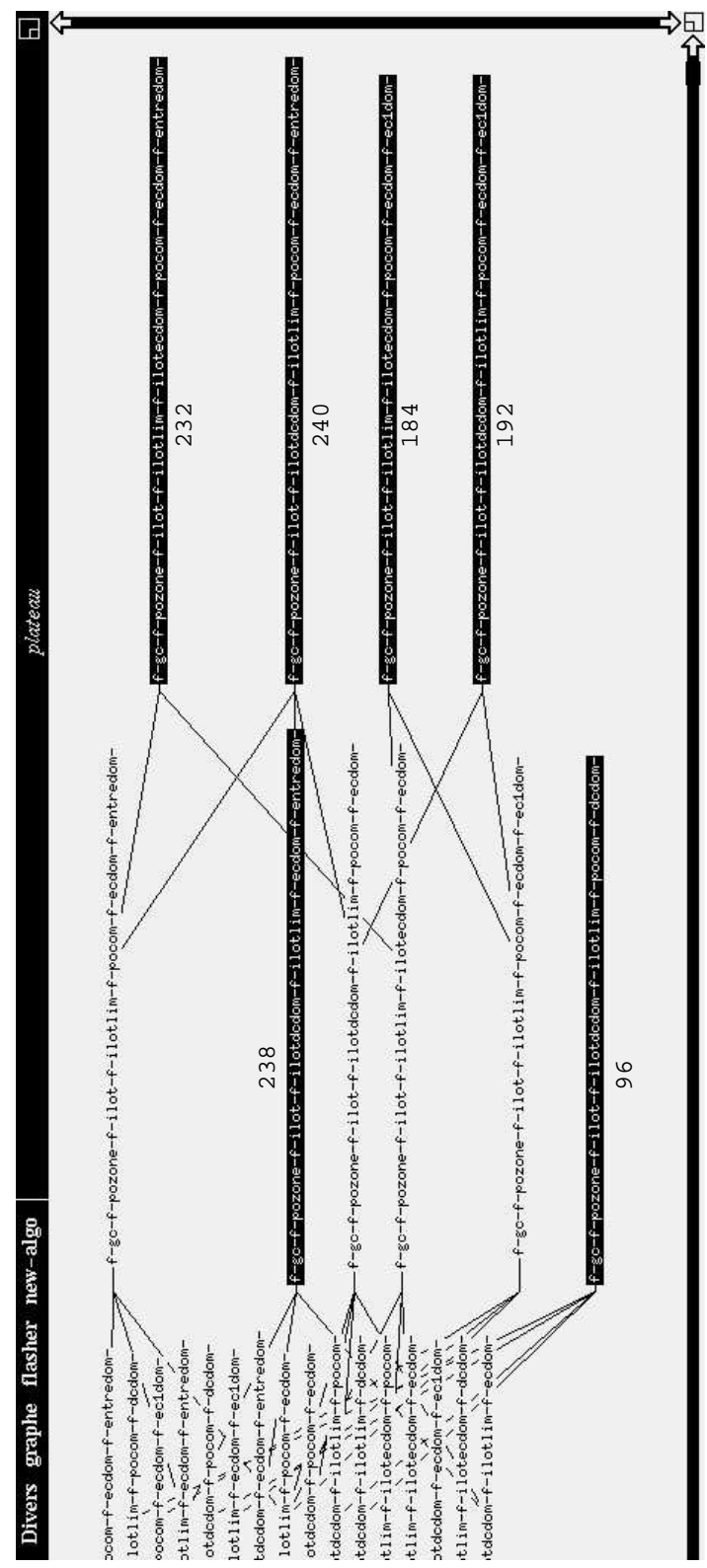

Figure 13: A part of the lattice representing the landscape models. 
TERRITORY-EC-FOREST class (see Figure 12). It checks therefore whether the instance t1 matches the properties of TERRITORY-EC-FOREST, i.e. $t$-ecn-f $=($ is-a.$E C)(a$. FOREST). It searches for forest entities in the neighborhood of $t 1$ in the image, and computes the topological relation between each forest entity and the territory, until it finds one forest entity externally connected to $t 1$. If it succeeds, then the $t-e c n-f$ property is verified and the instance $t 1$ is classified into the TERRITORY-EC-FOREST class. If it fails, the system tries to classify t 1 into another subclass of TERRITORY (e.g. TERRITORY-DC-FOREST). The classification process goes on down the hierarchy of spatial structures until all classes have been checked.

\subsection{Related work}

Our approach presents some similarities with the one used in the VEIL system for the recognition process of objects in an image [Price et al. 1994, Russ et al. 1996]: the domain model is described within hierarchies of concepts embedded in the LOOM system [MacGregor 1991, MacGregor and Brill 1992. The LOOM classifier is used to class the image objects into concepts according to the available information. The VEIL system combines both declarative knowledge and computational methods as in our system.

The work presented in Haarslev et al.1998] and Haarslev et al.1999] has also objectives that are similar to ours: it holds on qualitative spatial representation for managing map databases and spatial query processing. Spatial objects and relations are represented within the RACE system, that is based on description logics, and is extended to the polygon concrete domain, for taking into account spatial objects. Special modeling constructs can be used to represent topological relations as defined roles. Spatial reasoning relies on two main operations in description logics reasoning, namely consistency checking and classification. From the application point of view, regions are represented by polygons (elements of the concrete domain), and reasoning is carried on relations between polygons, for recognizing regions with specific characteristics and answering queries to a map database.

The objectives of this work and those of our work can be considered as complementary. The former work holds on polygons and is carried out in the context of description logics, with respect to the $R C C$ - 8 theory. In our work, we focus on the representation and reification of $R C C-8$ relations -relations correspond to concepts and not to roles-, within an object-based representation system, and on a lattice-based classification of spatial relations. The regions considered in our work are raster regions - and not vector regions-, but this does not make a fundamental difference between the two approaches from the reasoning point of view. The underlying reasoning mechanism is based in both cases on classification, and especially relation classification. Moreover, in [Möller and Wessel 1999], spatiotemporal default reasoning is introduced: a specific query completion problem is studied and default knowledge is used for completing and making more precise queries. This is still a complementary view with respect to our work. In our approach, classification and default knowledge represented within the classes are used to allow classification-based reasoning on spatial relation

\footnotetext{
${ }^{3}$ Forests cover a large area in Lorraine and are related to agriculture in many ways.
} 
and concepts. However, a more theoretical study of our approach has still to be carried out, as it is done in [Möller and Wessel 1999].

\section{Evaluation and research perspectives}

The system has been used on land-use maps representing a part of the Lorraine region (East of France) in 1990 Bachacou 1995. It has produced maps where the village territories are classified according to the landscape models of the agronomists. About $25 \%$ of the territories are misclassified or not classified: the first ones show that the lanscape models must be made more precise. The second ones are territories that cannot be classified according to the models. Actually, the experts are very interested both in the village territories that are classified and in those that are not classified. The first ones confirm and generalize their knowledge which is acquired from field studies. The last ones are 'special' territories that can indicate changes of the land use and thus that have to be further investigated. Besides, the system is still a prototype under development. Only partial evaluations have been performed until now, and a real-sized evaluation of the system remains to be done on other regions and dates (partial results are reported in Mangelinck 1998). By now, the present results of the system have indicated in which ways researches must be lead, for example by adding other spatial relations to describe the models (distance, orientation, and extended topology) and by refining the indices that describe the spatial entities.

The system and the associated research work can be enhanced and continued in a number of directions:

- The lattices considered above rely on the Galois connection theory. They are well adapted to the computation of relations, considering vector or raster data (images or geographic information systems). The choice of a set of computational primitives (two have been studied here, but other could be taken into account, such as the one proposed in [Ligozat 1999]) and then of the corresponding lattice $\mathcal{T}_{\mathcal{G}}$ or $\mathcal{T}_{\mathcal{G}}$ ' depends on the characteristics of the problem to be solved, as well as on the concrete definition of the boundaries, which is different if raster or vector data are used. This choice should rely on precise guidelines.

- The spatial domain contains regions which are not necessarily internally connected, in order to solve the planar realizibility problem Grigni et al.1995. However, this lead to another problem about the partial overlap relation $(P O(x, y))$ that may hold whereas the regions have no common boundary point $(N A(x, y))$. In the general case, $P O$ can be checked, using the conditions $D x, D y$, and $O$. But when $N A(x, y)$ has been already checked on two regions, then $P O(x, y)$ can not be inferred in the $\mathcal{T}_{\mathcal{G}}$ lattice: $(D x \wedge D y \wedge O \wedge N A)(x, y) \rightarrow \perp(x, y)$. In the future, it could thus be useful to extend the lattice by including two kinds of partial overlap, according to the boundary overlap. 
- In order to reason with spatial relations, it is better to exploit a complemented lattice such as $\mathcal{T}_{\mathcal{P}}$, or, at least, a lattice including all the relations of the composition table. A convenient compromise between relation computation and reasoning is to extend the Galois lattice $\mathcal{T}_{\mathcal{G}}$ in order to include the lacking relations. This leads to the lattice $\mathcal{T}_{\mathcal{G E}}$, where some elements are not computable but where all compositions of the base relations are present.

- Lattices such as $\mathcal{T}_{\mathcal{P}}$ are frequently used in systems based on the mereotopology for representing spatial knowledge and for spatial reasoning [Randell and Cohn 1992]. By contrast, lattices combining computation and reasoning purposes, such as the lattices $\mathcal{T}_{\mathcal{G}}$ and $\mathcal{T}_{\mathcal{G}}$, have not yet been used in the field of spatial representation and reasoning (at least to our knowledge). In addition to this "double competence", these two lattices have the main advantage of being minimal in terms of memory space (34 elements for $\mathcal{T}_{\mathcal{G}}$ versus 256 elements for $\mathcal{T}_{\mathcal{P}}$ ) and of being easily extensible: indeed, it is possible to modify, extend or reduce the set of computational primitives or the set of base relations without loosing the properties associated to the Galois connection theory.

- Lattices are well adapted to classification-based reasoning. For instance, the classification mechanism that we have developed for our application manages two related lattices: the first one is a lattice of relation classes based on $\mathcal{T}_{\mathcal{G}}$, and the second one is a lattice of classes representing the models of spatial structures. In this context, hierarchical case-based reasoning for interpretation and prediction purposes is a natural extension of classification-based reasoning (see for example Lieber and Napoli 1996. Lieber and Napoli 1998).

- A last word has to be said about the reification of relations in an object-based representation system. Actually, we have used an ad hoc implementation Mangelinck 1998. Le Ber et al.1999. A more general reification model has still to be studied, allowing to compose different types of relations and take advantage of these relations: inheritance of properties, representation of context, quantification, etc.

\section{Conclusion}

In this paper we have presented an original study for qualitative spatial representation and reasoning with topological relations. We have presented three lattices that can be used to compute and infer topological relations on raster images or data in geographic

information systems. We have used the base relations of the $R C C-8$ theory and a set of computational primitives on raster images and integrated them in a same lattice thanks to the Galois connection theory. We have shown that this lattice, named $\mathcal{T}_{\mathcal{G}}$, provides interesting properties for both computing and inferring relations, whereas the lattice based on the $\mathcal{B}$ subsets, named $\mathcal{T}_{\mathcal{P}}$, is well adapted for reasoning. We have extended $\mathcal{T}_{\mathcal{G}}$ into a lattice $\mathcal{T}_{\mathcal{G E}}$ in order to improve its reasoning capabilities. Finally we have compared $\mathcal{T}_{\mathcal{G}}$ with 
a fourth lattice, named $\mathcal{T}_{\mathcal{G}}$, built from the Galois connection between the base relations and the computational primitives proposed in [Egenhofer 1989.

This work has been done in the context of the design of a knowledge-based system for helping agricultural landscape analysis. The aim of this project is to automatically match spatial structures on land-use maps with landscape models defined by agronomists. These models are expressed as sets of spatial entities linked with qualitative spatial relations. The matching problem can be expressed in the framework of an object-based knowledge representation system, as a problem of instance classification, where the spatial structures appearing on the maps are instances and the landscape models are classes. Actually, the lattices $\mathcal{T}_{\mathcal{G}}$ and $\mathcal{T}_{\mathcal{G E}}$ have been implemented within the frame-based representation system Y3 and used for the classification of spatial structures.

For the future, other spatial relations such as qualitative distance relations and orientation relations are planned to be added to the system, leading to new research problems both in the theoretical field of space representation and in the field of object-based knowledge representation systems.

$\mathrm{RR} \mathrm{n}^{\circ} 4321$ 


\section{Appendix}

\section{Composition of the base relations of the $R C C-8$ theory}

There are 12 disjunctions of relations in the transitivity table of $\mathcal{B}$ (Table 6):

$$
\begin{array}{r}
D C \vee E C \vee P O \vee T P P \vee N T P P \\
D C \vee E C \vee P O \vee T P P^{-1} \vee N T P P^{-1} \\
D C \vee E C \vee P O \vee T P P \vee T P P^{-1} \vee E Q \\
D C \vee E C \\
T P P \vee N T P P \\
T P P^{-1} \vee N T P P^{-1} \\
P O \vee T P P \vee N T P P \vee T P P^{-1} \vee N T P P^{-1} \vee E Q \\
P O \vee T P P^{-1} \vee N T P P^{-1} \\
P O \vee T P P \vee N T P P \\
P O \vee T P P \vee T P P^{-1} \vee E Q \\
E C \vee P O \vee T P P^{-1} \vee N T P P^{-1} \\
E C \vee P O \vee T P P \vee N T P P
\end{array}
$$

\section{The elements of the lattice $\mathcal{T}_{\mathcal{G}}$}

The 32 elements of the lattice (except the top and bottom elements) are described in the Table 11

\section{Comparing the Galois lattices $\mathcal{T}_{\mathcal{G}}$ and $\mathcal{T}_{\mathcal{G}}$ '}

The table 12 shows the number of conditions used for computing each relation that is expressed in the lattices $\mathcal{T}_{\mathcal{G}}$ and $\mathcal{T}_{\mathcal{G}}$ '. The first column gives the sets of relations $R$ expressed in the lattices. The two following columns give the names of the corresponding elements in the two lattices (E' and E). The two last columns give the number $\left(n^{\prime}, n\right)$ of conditions used to check the relations (i.e. the cardinality of the minimal set of conditions, see paragraph 3.4.3). The eight base relations are listed in the first frame. The other relations common to the two lattices are represented in the second frame. The third and fourth frames list respectively the relations that are only represented in $\mathcal{T}_{\mathcal{G}}$, or in $\mathcal{T}_{\mathcal{G}}$.

\section{Definitions and notations}

The definitions are based on Barbut and Monjardet 1970. 


\begin{tabular}{|c|c|c|}
\hline$R$ & $C$ & $\mathrm{E}$ \\
\hline$\{D C\}$ & $\{D R, N A, D x, D y\}$ & DC \\
\hline$\{E C\}$ & $\{D R, A, D x, D y\}$ & $\mathrm{EC}$ \\
\hline$\{P O\}$ & $\{O, D x, D y, A\}$ & PO \\
\hline$\left\{T P P^{-1}\right\}$ & $\left\{O, P^{-1}, D x, A\right\}$ & $\mathrm{TPP}^{-1}$ \\
\hline$\{T P P\}$ & $\{O, P, D y, A\}$ & TPP \\
\hline$\{N T P P\}$ & $\{O, P, D y, N A\}$ & NTPP \\
\hline$\left\{N T P P^{-1}\right\}$ & $\left\{O, P^{-1}, D x, N A\right\}$ & NTPP $^{-1}$ \\
\hline$\{E Q\}$ & $\left\{O, P, P^{-1}, A\right\}$ & EQ \\
\hline$\{D C, N T T P\}$ & $\{N A, D y\}$ & DyetNA \\
\hline$\left\{D C, N T P P^{-1}\right\}$ & $\{N A, D x\}$ & DxetNA \\
\hline$\left\{P O, T P P^{-1}\right\}$ & $\{O, D x, A\}$ & DetAetDx \\
\hline$\{P O, T P P\}$ & $\{O, D y, A\}$ & DetAetDy \\
\hline$\{T P P, N T P P\}$ & $\{O, P, D y\}$ & PP \\
\hline$\{T P P, E Q\}$ & $\{O, P, A\}$ & TP \\
\hline$\left\{T P P^{-1}, N T P P^{-1}\right\}$ & $\left\{O, P^{-1}, D x\right\}$ & $\mathrm{PP}^{-1}$ \\
\hline$\left\{T P P^{-1}, E Q\right\}$ & $\left\{O, P^{-1}, A\right\}$ & $\mathrm{TP}^{-1}$ \\
\hline$\left\{N T P P, N T P P^{-1}\right\}$ & $\{O, N A\}$ & DetNA \\
\hline$\{P O, T P P, N T P P\}$ & $\{O, D y\}$ & Dyet0 \\
\hline$\left\{P O, T P P^{-1}, N T P P^{-1}\right\}$ & $\{O, D x\}$ & Dxet0 \\
\hline$\left\{T P P, T P P^{-1}, E Q, P O\right\}$ & $\{O, A\}$ & DetA \\
\hline$\{E C, P O\}$ & $\{A, D x, D y\}$ & DxetDyetA \\
\hline$\left\{E C, P O, T P P^{-1}\right\}$ & $\{A, D x\}$ & DxetA \\
\hline$\{E C, P O, T P P\}$ & $\{A, D y\}$ & DyetA \\
\hline$\{E C, P O, D C\}$ & $\{D x, D y\}$ & DxetDy \\
\hline$\{T P P, N T P P, E Q\}$ & $\{O, P\}$ & $\bar{P}$ \\
\hline$\left\{T P P^{-1}, N T P P^{-1}, E Q\right\}$ & $\left\{O, P^{-1}\right\}$ & $\mathrm{P}^{-1}$ \\
\hline$\{D C, E C\}$ & $\{D R, D x, D y\}$ & $\mathrm{DR}$ \\
\hline$\left\{D C, N T P P, N T P P^{-1}\right\}$ & $\{N A\}$ & NA \\
\hline$\left\{T P P, T P P^{-1}, E Q, P O, E C\right\}$ & $\{A\}$ & A \\
\hline$\left\{T P P, N T P P, T P P^{-1}, N T P P^{-1}, E Q, P O\right\}$ & $\{O\}$ & 0 \\
\hline$\left\{E C, P O, D C, T P P^{-1}, N T P P^{-1}\right\}$ & $\{D x\}$ & $\mathrm{Dx}$ \\
\hline$\{E C, P O, D C, T P P, N T P P\}$ & $\{D y\}$ & Dy \\
\hline
\end{tabular}

Table 11: The elements of the lattice $\mathcal{T}_{\mathcal{G}}: \mathrm{E}$ is the name of the element, $R$ the subset of $\mathcal{B}$, $C$ the subset of $C M-8$. 


\begin{tabular}{|c|c|c|c|c|}
\hline$R$ & $\mathrm{E}^{\prime}$ & $E$ & $n^{\prime}$ & $n$ \\
\hline$\{D C\}$ & $\overline{\mathrm{DC}}$ & $\overline{\mathrm{DC}}$ & 2 & 2 \\
\hline$\{E C\}$ & EC & EC & 2 & 2 \\
\hline$\{P O\}$ & PO & PO & 2 & 3 \\
\hline$\left\{T P P^{-1}\right\}$ & $\mathrm{TPP}^{-1}$ & $\mathrm{TPP}^{-1}$ & 3 & 3 \\
\hline$\{T P P\}$ & TPP & TPP & 3 & 3 \\
\hline$\{N T P P\}$ & NTPP & NTPP & 2 & 2 \\
\hline$\left\{N T P P^{-1}\right\}$ & $\mathrm{NTPP}^{-1}$ & $\mathrm{NTPP}^{-1}$ & 2 & 2 \\
\hline$\{E Q\}$ & $\mathrm{EQ}$ & $\mathrm{EQ}$ & 3 & 2 \\
\hline$\{D C, E C\}$ & $\mathrm{DR}$ & $\mathrm{DR}$ & 1 & 1 \\
\hline$\{D C, N T T P\}$ & NOFetNA & DyetNA & 2 & 2 \\
\hline$\left\{D C, N T P P^{-1}\right\}$ & NFOetNA & DxetNA & 2 & 2 \\
\hline$\left\{P O, T P P^{-1}\right\}$ & OFetA & DetAetDx & 2 & 3 \\
\hline$\{P O, T P P\}$ & FOetA & DetAetDy & 2 & 3 \\
\hline$\{T P P, N T P P\}$ & PP & PP & 2 & 2 \\
\hline$\{T P P, E Q\}$ & TP & $\mathrm{TP}$ & 3 & 2 \\
\hline$\left\{T P P^{-1}, N T P P^{-1}\right\}$ & $\mathrm{PP}^{-1}$ & $P P^{-1}$ & 2 & 2 \\
\hline$\left\{T P P^{-1}, E Q\right\}$ & $\mathrm{TP}^{-1}$ & $\mathrm{TP}^{-1}$ & 3 & 2 \\
\hline$\left\{N T P P, N T P P^{-1}\right\}$ & OetNA & DetNA & 2 & 2 \\
\hline$\left\{D C, N T P P, N T P P^{-1}\right\}$ & NA & $\mathrm{NA}$ & 1 & 1 \\
\hline$\{P O, T P P, N T P P\}$ & FO & Dyet0 & 1 & 2 \\
\hline$\left\{P O, T P P^{-1}, N T P P^{-1}\right\}$ & $\mathrm{OF}$ & Dxet0 & 1 & 2 \\
\hline$\{T P P, N T P P, E Q\}$ & $\mathrm{P}$ & $\mathrm{P}$ & 2 & 1 \\
\hline$\left\{T P P^{-1}, N T P P^{-1}, E Q\right\}$ & $\mathrm{P}^{-1}$ & $\mathrm{P}^{-1}$ & 2 & 1 \\
\hline$\left\{T P P, T P P^{-1}, E Q, P O\right\}$ & OetA & DetA & 2 & 2 \\
\hline$\left\{T P P, T P P^{-1}, E Q, P O, E C\right\}$ & A & A & 1 & 1 \\
\hline$\left\{T P P, N T P P, T P P^{-1}, N T P P^{-1}, E Q, P O\right\}$ & 0 & 0 & 1 & 1 \\
\hline$\{E C, E Q\}$ & NFOetNOFetA & & 3 & \\
\hline$\{E C, E Q, T P P\}$ & AetNOF & & 2 & \\
\hline$\left\{E C, E Q, T P P^{-1}\right\}$ & AetNFO & & 2 & \\
\hline$\{E C, E Q, D C\}$ & NFOetNOF & & 2 & \\
\hline$\{E C, E Q, D C, T P P, N T P P\}$ & NOF & & 1 & \\
\hline$\left\{E C, E Q, D C, T P P^{-1}, N T P P^{-1}\right\}$ & NFO & & 1 & \\
\hline$\{E C, P O\}$ & & DxetDyetA & & 3 \\
\hline$\left\{E C, P O, T P P^{-1}\right\}$ & & DxetA & & 2 \\
\hline$\{E C, P O, T P P\}$ & & DyetA & & 2 \\
\hline$\{E C, P O, D C\}$ & & DxetDy & & 2 \\
\hline$\left\{E C, P O, D C, T P P^{-1}, N T P P^{-1}\right\}$ & & $\mathrm{Dx}$ & & 1 \\
\hline$\{E C, P O, D C, T P P, N T P P\}$ & & Dy & & 1 \\
\hline
\end{tabular}

Table 12: Comparing the number of conditions used to check the relations in the Galois lattices $\mathcal{T}_{\mathcal{G}}$ and $\mathcal{T}_{\mathcal{G}}$ ': E' and $\mathrm{E}$ are the names of the elements in the two lattices, $n^{\prime}$ and $n$ the number of conditions used to check a relation. 
Partial ordering. $X$ is a non empty set, $\leq$ is a binary relation defined on $X,(X, \leq)$ is a partial ordering if-f:

1. $\leq$ is reflexive: $\forall x \in X, x \leq x$,

2. $\leq$ is antisymmetric: $\forall x, y \in X,(x \leq y$ et $y \leq x) \Rightarrow(x=y)$,

3. $\leq$ is transitive: $\forall x, y, z \in X,(x \leq y$ et $y \leq z) \Rightarrow(x \leq z)$.

Infimum or glb. $(X, \leq)$ is a partial ordering, $A$ is a subset of $X, m$ is an element of $X$, $m$ is an infimum of $A$ if-f:

- $\forall a \in A,(m \leq a)$,

- $\forall c \in X,(\forall a \in A, c \leq a) \Rightarrow(c \leq m)$.

Supremum or lub. $(X, \leq)$ is a partial ordering, $A$ is a subset of $X, m$ is an element of $X, m$ is a supremum of $A$ if-f:

- $\forall a \in A,(a \leq M)$,

- $\forall c \in X,(\forall a \in A, a \leq c) \Rightarrow(M \leq c)$.

Lattice. A lattice $\langle X, \leq, \frown, \smile\rangle$ is a partial ordering $(X, \leq)$ where each pair $\{x, y\} \in X^{2}$ has a supremum $\smile$ and an infimum $\neg$.

Complemented lattice. $\langle X, \leq, \frown, \smile\rangle$ is a lattice with a bottom element $O$ and a top element $I$. $X$ is said to be complemented if all the elements of $X$ have a complement element in $X$ that is if:

$$
\forall x \in X, \exists y \in X:(x \smile y=I \text { et } x \frown y=O)
$$

Distributive lattice. A lattice $\langle X, \leq, \frown, \smile\rangle$ is said to be distributive if it obeys to the following expressions for all the triplets $(x, y, z)$ :

$$
\begin{array}{ll}
(\mathrm{D} \frown) & x \frown(y \smile z)=(x \frown y) \smile(x \frown z) \\
(\mathrm{D} \smile) & x \smile(y \frown z)=(x \smile y) \frown(x \smile z) \\
(\mathrm{M} d) & (x \frown y) \smile(y \frown z) \smile(z \frown x)=(x \smile y) \frown(y \smile z) \frown(z \smile y)
\end{array}
$$

It can be demonstrated that any of the three expressions implies the two others.

\footnotetext{
${ }^{4}$ The infimum and the supremum are generally denoted with $\wedge$ and $\vee$ but we rather use these symbols to denote the logical conjunction and disjunction.
} 
Closure. $h$ is an application in a ordered set $(E, \leq), h$ is a closure operator if it respects the three following properties:

- $\forall x, \forall y, x \leq y \Rightarrow h(x) \leq h(y)$ ( $h$ is monotone)

- $\forall x, x \leq h(x)$ ( $h$ is extensive)

- $\forall x, h \circ h(x)=h(x)$ ( $h$ is idempotent $)$

An element of $\mathrm{E}$ is closed with respect to $h$ if it is equal to its closure by $h(x=h(x))$.

\section{Proofs}

We first admit the following hypotheses:

(1) The relations of the $R C C$-8 theory are exhaustive and mutually exclusive, i.e. for all pair $(x, y) \in \mathcal{D}^{2}$, there is one and only one relation of $\mathcal{B}$ that holds: $\forall(x, y) \in \mathcal{D}^{2}, \exists r \in$ $\mathcal{B}: r(x, y)$ and $\forall r^{\prime} \in \mathcal{B}, r^{\prime} \neq r \rightarrow \neg r^{\prime}(x, y)$

(2) A relation of $\mathcal{B}$ does imply a condition of $C M-8$ or its negation: $\forall r \in \mathcal{B}, \forall c \in$ $C M-8, r \notin \mathcal{C}^{\prime}(c) \leftrightarrow r \in \mathcal{C}^{\prime}(\neg c)$ or $\mathcal{C}^{\prime}(c) \cup \mathcal{C}^{\prime}(\neg c)=\mathcal{B}$

(3) For all relation of $\mathcal{B}$, there is no other relation that implies the same conditions of $C M-8: \forall r \in \mathcal{B}, \neg \exists r^{\prime} \in \mathcal{B}, \mathcal{C}(r) \subseteq \mathcal{C}\left(r^{\prime}\right)$

Lemma 1 For all subset $R$ of $\mathcal{B}, f(R)=f(\bar{R})$ where $\bar{R}=h(R)$ (that is, all subset $C=f(R)$ of $C M-8$ is closed).

Proof: $f(R)=\bigcap_{r \in R} \mathcal{C}(r)$ and $f(\bar{R})=\bigcap_{r \in \bar{R}} \mathcal{C}(r)$

$R \subseteq \bar{R}$ (extensiviy of $h$ ) then $f(\bar{R}) \subseteq f(R)$

Conversely: $f(\bar{R})=f \circ h(R)=f \circ g \circ f(R)=h^{\prime} \circ f(R)$

then $f(R) \subseteq f(\bar{R})$ (extensiviy of $h^{\prime}$ ).

Lemma 2 For all subset $C$ of $C M-8, g(C)=g(\bar{C})$ où $\bar{C}=h^{\prime}(C)$ (that is, all subset $R=g(C)$ of $\mathcal{B}$ is closed).

Proof: $g(C)=\bigcap_{c \in C} \mathcal{C}^{\prime}(c)$ and $g(\bar{C})=\bigcap_{c \in \bar{C}} \mathcal{C}^{\prime}(c)$

$C \subseteq \bar{C}$ (extensiviy of $h^{\prime}$ ) then $g(\bar{C}) \subseteq f(C)$

Conversely: $g(\bar{C})=g \circ h^{\prime}(C)=g \circ f \circ g(C)=h \circ g(C)$ then $g(C) \subseteq g(\bar{C})$ (extensiviy of $h$ ).

Lemma 3 For all subset $R$ of $\mathcal{B}$, the disjunction of the relations of $R$ implies the conjunction of the conditions of $f(R)$, i.e.:

$$
\forall(x, y) \in \mathcal{D}^{2}, \bigvee_{r \in R} r(x, y) \rightarrow \bigwedge_{c \in f(R)} c(x, y)
$$


Proof: For all $c \in f(R)$ :

$(c \in f(R)) \leftrightarrow\left(c \in \bigcap_{r \in R} \mathcal{C}(r)\right) \rightarrow\left(\forall r \in R, \forall(x, y) \in \mathcal{D}^{2}, r(x, y) \rightarrow c(x, y)\right)$.

Then: $\forall r \in R, \forall(x, y) \in \mathcal{D}^{2}, r(x, y) \rightarrow \bigwedge_{c \in f(R)} c(x, y)$.

Then: $\forall(x, y) \in \mathcal{D}^{2}, \bigvee_{r \in R} r(x, y) \rightarrow \bigwedge_{c \in f(R)} c(x, y)$.

Lemma 4 For all subset $C$ of $C M-8$, the conjunction of the conditions of $C$ implies the disjunction of the relations of $g(C)$, i.e.:

$$
\forall(x, y) \in \mathcal{D}^{2}, \bigwedge_{c \in C} c(x, y) \rightarrow \bigvee_{r \in g(C)} r(x, y)
$$

Proof: we prove the contraposition: if no relation of $g(C)$ holds for a pair $(x, y)$ then at least one condition of $C$ does not hold for this pair.

Suppose $g(C) \neq \mathcal{B}$.

$\left(\exists(x, y) \in \mathcal{D}^{2}, \forall r \in g(C), \neg r(x, y)\right) \rightarrow \exists r^{\prime} \in(\mathcal{B}-g(C)), r^{\prime}(x, y)$ (cf. Hypothesis (1)).

$r^{\prime} \notin g(C)=\bigcap_{c \in C} \mathcal{C}^{\prime}(c)$ then: $\exists c \in C, r^{\prime} \notin \mathcal{C}^{\prime}(c)$.

According to the hypothesis $(2), r^{\prime}(x, y)$ implies the negation of $c(x, y)$. Finally:

$$
\forall(R, C) \in \mathcal{E}_{\mathcal{G}}, \forall(x, y) \in \mathcal{D}^{2}, \neg \bigvee_{r \in R} r(x, y) \rightarrow \neg \bigwedge_{c \in C} c(x, y)
$$

If $g(C)=\mathcal{B}$, then the disjunction of $\mathcal{B}$ elements holds (cf. Hypothesis (1)).

Lemma 5 For all subset $C$ of $C M-8$, the conjunction of the conditions of $C$ is equivalent to the conjunction of the conditions of the $h^{\prime}$-closure of $C$ :

$$
\forall(x, y) \in \mathcal{D}^{2}, \bigwedge_{c \in C} c(x, y) \leftrightarrow \bigwedge_{c \in h^{\prime}(C)} c(x, y)
$$

Proof: We denote $\bar{C}=h^{\prime}(C)$.

$h^{\prime}$ is monotonously growing then $\forall C \in 2^{C M-8}, C \subseteq \bar{C}$.

Then $\forall(x, y) \in \mathcal{D}^{2}, \bigwedge_{c \in \bar{C}} c(x, y) \rightarrow \bigwedge_{c \in C} c(x, y)$.

Conversely, according to the lemmas 3 and 4

$\forall(x, y) \in \mathcal{D}^{2}, \bigwedge_{c \in C} c(x, y) \rightarrow \bigvee_{r \in g(C)} r(x, y) \rightarrow \bigwedge_{c \in f \circ g(C)} c(x, y)=\bigwedge_{c \in \bar{C}} c(x, y)$.

Proof of the property 1; If a pair $E=(C, R)$ is an element of the lattice $\mathcal{T}_{\mathcal{G}}$ then the disjunction of the elements of $R$ is equivalent to the conjunction of the elements of $C$. 
According to the definition of the Galois lattice, $(C, R) \in \mathcal{E}_{\mathcal{G}} \leftrightarrow C=f(R)$ and $R=f^{\prime}(C)$.

Direct implication: it is directly based on the lemma 3 and on $C=f(R)$.

$\forall(R, C) \in \mathcal{E}_{\mathcal{G}}, \forall(x, y) \in \mathcal{D}^{2}, \bigvee_{r \in R} r(x, y) \rightarrow \bigwedge_{c \in C} c(x, y)$

Converse implication: it is directly based on the lemma 4 and on $R=g(C)$.

$\forall(R, C) \in \mathcal{E}_{\mathcal{G}}, \forall(x, y) \in \mathcal{D}^{2}, \bigwedge_{c \in C} c(x, y) \rightarrow \bigvee_{r \in R} r(x, y)$

Proof of the property 2, $R$ is a subset of $\mathcal{B}$ and $C$ is a subset of $C M-8$. If the conjunction of the $C$ elements is equivalent to the disjunction of the $R$ elements, then the pair $(\bar{C}, R)$ is an element of the lattice $\mathcal{T}_{\mathcal{G}}$.

According to the lemma 5 and the hypothesis:

$\forall(x, y) \in \mathcal{D}^{2}, \bigvee_{r \in R} r(x, y) \leftrightarrow \bigwedge_{c \in C} c(x, y) \leftrightarrow \bigwedge_{c \in \bar{C}} c(x, y)$

$(\bar{C}, g(\bar{C}))$ is a element of the lattice (definition of the Galois lattice).

$g(\bar{C})$ is denoted with $R^{\prime}$. The aim is to prove $R=R^{\prime}$.

According to the property 1 .

$$
\left(\bar{C}, R^{\prime}\right) \in \mathcal{T}_{\mathcal{G}} \rightarrow\left(\forall(x, y) \in \mathcal{D}^{2}, \bigwedge_{c \in \bar{C}} c(x, y) \leftrightarrow \bigvee_{r \in R^{\prime}} r(x, y)\right)
$$

Hence $\forall(x, y) \in \mathcal{D}^{2}, \bigvee_{r \in R} r(x, y) \leftrightarrow \bigvee_{r \in R^{\prime}} r(x, y)$

For all relation $r \in R,(x, y)$ is a pair of $\mathcal{D}^{2}$ for which $r$ holds: then it exists a relation $r^{\prime} \in R^{\prime}$ that holds for $(x, y)$. According to the hypothesis (1), $r=r^{\prime}$. Conversely, for all relation $r^{\prime} \in R^{\prime}$, it exists a relation $r \in R$ such that $r=r^{\prime}$. The two sets are equal.

Proof of the property 3 For all element $r$ of $\mathcal{B},(f(\{r\}),\{r\})$ is an element of the lattice $\mathcal{T}_{\mathcal{G}}$.

This property can be proved in different ways. The first one is to observe (cf. Tablel7) that any relation of $\mathcal{B}$ is equivalent to a conjunction of four conditions of $C M-8 . C_{r}$ denotes the subset of the four conditions. According to the property 2 $\left(\bar{C}_{r},\{r\}\right)$ is an element of the lattice. Hence $\{r\}$ is closed for $h$ and $\bar{C}_{r}=f(\{r\})$.

The second way is to prove directly that $\{r\}$ is closed by $h$. Suppose it is false, i.e. it exists a relation $r^{\prime} \neq r$ that belongs to $h(\{r\})$.

$h(\{r\})=g \circ f(\{r\})=g(\mathcal{C}(r))=\bigcap_{c \in C(r)} \mathcal{C}^{\prime}(c)$.

$r^{\prime} \in h(\{r\}) \rightarrow\left(\forall c \in \mathcal{C}(r), \forall(x, y), r^{\prime}(x, y) \rightarrow c(x, y)\right)$.

$r^{\prime} \in h(\{r\}) \rightarrow \mathcal{C}(r) \subseteq \mathcal{C}\left(r^{\prime}\right)$ which is impossible according to the hypothesis (3).

INRIA 
Proof of the property 4; Any condition $c$ of $C M-8$ can be associated to an element $\mathrm{E}_{\mathrm{c}}=(C, R)$, where $C=h^{\prime}(\{c\})$ and $R=g(C)=g(\{c\})$. This element is singular for each condition.

First, according to the lemma [5 any condition $c$ of $C M-8$ is equivalent to the conjunction of the conditions of its $h^{\prime}$-closure. Hence: $\forall(x, y) \in \mathcal{D}^{2}, c(x, y) \leftrightarrow \bigwedge_{c \in \overline{\{c\}}} c(x, y)$

$\overline{\{c\}}=h^{\prime}(\{c\})$ being closed by $h^{\prime}$, the element $\mathrm{E}_{\mathrm{c}}=(\overline{\{c\}}, g(\overline{\{c\}}))$ is an element of the lattice $\mathcal{T}_{\mathcal{G}} ; g(\overline{\{c\}})=g(\{c\})$ according to the lemma 2] Finally, according to the property 1

$$
\forall(x, y) \in \mathcal{D}^{2}, c(x, y) \leftrightarrow \bigwedge_{c \in \overline{\{c\}}} c(x, y) \leftrightarrow \bigvee_{r \in g(\{c\})} r(x, y)
$$

The element $E_{c}$ is singular for each condition since the conditions are pairwise not equivalent.

Proof of the property 5 . An element $E=(C, R)$ of the lattice is the $g l b$ of two elements $E_{1}=\left(C_{1}, R_{1}\right)$ and $E_{2}=\left(C_{2}, R_{2}\right)$ if and only if the relation $\pi_{r}(E)$ is equal to the conjunction of the two relations $\left(\pi_{r}\left(E_{1}\right)\right)$ and of $R_{2}\left(\pi_{r}\left(E_{2}\right)\right)$ :

$$
\left(\mathrm{E}=\mathrm{E}_{1} \frown \mathrm{E}_{2}\right) \leftrightarrow\left(\forall(x, y) \in \mathcal{D}^{2}, \pi_{r}(\mathrm{E})(\mathrm{x}, \mathrm{y}) \leftrightarrow \pi_{\mathrm{r}}\left(\mathrm{E}_{1}\right)(\mathrm{x}, \mathrm{y}) \wedge \pi_{\mathrm{r}}\left(\mathrm{E}_{2}\right)(\mathrm{x}, \mathrm{y})\right)
$$

The $g l b$ of two elements of the lattice is defined as follows:

$\left(C_{1}, R_{1}\right) \frown\left(C_{2}, R_{2}\right)=\left(h^{\prime}\left(C_{1} \cup C_{2}\right), R_{1} \cap R_{2}\right)$.

Direct implication: $E$ is the $g l b$ of the elements $E_{1}$ and $E_{2}$.

Suppose $\bigvee_{r \in R} r(x, y)$ holds for a pair of objects $(x, y) \in \mathcal{D}^{2}$.

Hence: $\exists r \in R, r(x, y)$

$\left(r \in R_{1} \cap R_{2}\right.$ and $\left.r(x, y)\right) \rightarrow \bigvee_{r \in R_{1}} r(x, y) \wedge \bigvee_{r \in R_{2}} r(x, y)$.

Conversely, suppose $\bigvee_{r \in R_{1}} r(x, y) \wedge \bigvee_{r \in R_{2}} r(x, y)$ holds for a pair of objects $(x, y)$.

Hence $\exists r_{1} \in R_{1}, \exists r_{2} \in R_{2}, r_{1}(x, y) \wedge r_{2}(x, y)$.

According to the hypothesis (2), $r_{1}=r_{2}$, hence $r_{1} \in R_{1} \cap R_{2}$ and $\bigvee_{r \in R} r(x, y)$ holds.

Converse implication: $E, E_{1}$ and $E_{2}$ are three elements of the lattice such that:

$$
\bigvee_{r \in R} r(x, y) \leftrightarrow \bigvee_{r \in R_{1}} r(x, y) \wedge \bigvee_{r \in R_{2}} r(x, y)
$$

Proving $R=R_{1} \cap R_{2}$ is enough; $C=h^{\prime}\left(C_{1} \cup C_{2}\right)$ can be deduced immediately by $C=f(R)$ and $g\left(C_{1}\right) \cap g\left(C_{2}\right)=g\left(C_{1} \cup C_{2}\right)$.

Let be any relation $r \in R$ and a pair $(x, y) \in \mathcal{D}^{2}$ such that $r(x, y)$ holds. 
Hence $\bigvee_{r \in R} r(x, y)$ holds and thus $\bigvee_{r \in R_{1}} r(x, y) \wedge \bigvee_{r \in R_{2}} r(x, y)$ holds.

Hence $\exists r_{1} \in R_{1}, \exists r_{2} \in R_{2}, r_{1}(x, y) \wedge r_{2}(x, y)$.

According to the hypothesis (1), $r_{1}=r_{2}=r$ hence $R \subseteq R_{1} \cap R_{2}$.

Conversely, let be a relation $r \in R_{1} \cap R_{2}$ and a pair of objects $(x, y) \in \mathcal{D}^{2}$ such that $r(x, y)$ holds. The same reasoning leads to $R_{1} \cap R_{2} \subseteq R$.

Proof of the property [7, For any pair of elements of the lattice $\left(E_{1}, E_{2}\right)$, the $l u b$ $\mathrm{E}^{\prime}=\mathrm{E}_{1} \smile \mathrm{E}_{2}$ verifies that the disjunction of the relations $\pi_{r}\left(\mathrm{E}_{1}\right)$ and $\pi_{r}\left(\mathrm{E}_{2}\right)$ implies the relation $\pi_{r}\left(\mathrm{E}^{\prime}\right)$.

The $l u b$ of two elements of the lattice is defined as follows:

$\left(C_{1}, R_{1}\right) \smile\left(C_{2}, R_{2}\right)=\left(C_{1} \cap C_{2}, h\left(R_{1} \cup R_{2}\right)\right)$.

Let be $\mathrm{E}^{\prime}=\left(C^{\prime}, R^{\prime}\right)$ and $R^{\prime}=h\left(R_{1} \cup R_{2}\right) \supseteq R_{1} \cup R_{2}$

Hence:

$$
\left(\bigvee_{r \in R^{\prime}} r(x, y)=\bigvee_{r \in h\left(R_{1} \cup R_{2}\right)} r(x, y)\right) \leftarrow\left(\bigvee_{r \in R_{1} \cup R_{2}} r(x, y)=\bigvee_{r \in R_{1}} r(x, y) \vee \bigvee_{r \in R_{2}} r(x, y)\right)
$$




\section{References}

[Allen 1983] J.F. Allen. Maintaining Knowledge about Temporal Intervals. Communications of the ACM, 26(11):832-843, November 1983.

[Asher and Vieu 1995] N. Asher and L. Vieu. Toward a Geometry of Common Sense: A Semantic and a Complete Axiomatization of Mereotopology. In Proceedings of the Fourteenth International Joint Conference on Artificial Intelligence (IJCAI'95), pages 846-852, San Mateo, CA, 1995.

[Bachacou 1995] J. Bachacou. Map of the land use in Lorraine based on Landsat images, crop year 1989-90. Document, January 1995. Lab. d'Intelligence Artificielle et de Biométrie, INRA-Nancy, France.

[Barbut and Monjardet 1970] M. Barbut and B. Monjardet. Ordre et classification - Algèbre et combinatoire. Hachette, Paris, 1970.

[Benoît 1990] M. Benoît. La gestion territoriale de l'activité agricole dans un village lorrain. Mappemonde, (4):15-17, 1990.

[Carpineto and Romano 1993] C. Carpineto and G. Romano. Galois: An order-theoretic approach to conceptual clustering. In Proceedings of the 10th International Conference on Machine Learning (ICML'93), Amherst, pages 33-40. Morgan Kaufmann, 1993.

[Clarke 1981] B.L. Clarke. A calculus of individuals based on 'connection'. Notre Dame Journal of Formal Logic, 22(3):204-218, 1981.

[Clarke 1985] B. L. Clarke. Individuals and points. Notre Dame Journal of Formal Logic, 26(1):61-75, 1985 .

[Clementini et al. 1993] E. Clementini, P. Di Felice, and P. van Oosterom. A small set of formal topological relationships for end-user interaction. In D. J. Abel and B. C. Ooi, editors, Advances in Spatial Databases: Proceedings of the Third International Symposium, SSD'93, LNCS 692, pages 277-295, Singapore, June 23-25 1993. Springer Verlag.

[Cohn et al. 1993] A.G. Cohn, D. A. Randell, Z. Cui, and B. Bennet. Qualitative Spatial Reasoning and Representation. In P. Carrete and M.G. Singh, editors, Qualitative Reasoning and Decision Technologies, pages 513-522, Barcelona, June 1993.

[Cohn et al. 1997] A. G. Cohn, B. Bennett, J. Gooday, and N. M. Gotts. Representing and Reasoning with Qualitative Spatial Relations About Regions, chapter 4, pages 97-134. In Stock [Stock 1997], 1997.

[Cui et al. 1993] Z. Cui, A.G. Cohn, and D.A. Randell. Qualitative and Topological Relationships in Spatial Databases. In D. Abel and B.C. Ooi, editors, Advances in Spatial Databases: Proceedings of the Third International Symposium, SD'93, LNCS 692, pages 296-315. Springer-Verlag, 1993. 
[Davey and Priestley 1990] B.A. Davey and H.A. Priestley. Introduction to Lattices and Order. Cambridge University Press, Cambridge, UK, 1990.

[Deffontaines 1986] J.-P. Deffontaines. Un point de vue d'agronome sur le paysage. Une méthode d'analyse du paysage pour l'étude de l'activité agricole. In Lectures du paysage, pages $35-52$. Foucher, 1986. Coll. INRAP-Dijon.

[Deffontaines 1990] J.-P. Deffontaines. Organisation spatiale de l'activité agricole et développement d'une petite région lorraine. Mappemonde, (4):12-14, 1990.

[Ducournau 1991] R. Ducournau. Y3: YAFOOL, the object oriented language, 1991. SEMA Group.

[Egenhofer and Sharma 1993] M. J. Egenhofer and J. Sharma. Topological Relations Between Regions in $\Re^{2}$ and $Z^{2}$. In D. J. Abel and B. C. Ooi, editors, Advances in spatial databases: Proceedings of the Third International Symposium, SSD'93, LNCS 692, pages 316-336. Springer Verlag, 1993.

[Egenhofer 1989] M. J. Egenhofer. A Formal Definition of Binary Topological Relationships. In W. Litwin and H.J. Schek, editors, Foundations of data organization and algorithms, proceedings of the 3rd international conference, FODO 1989, LNCS 367, pages 457-472. Springer Verlag, 1989. Paris, juin.

[Frank 1997] A. U. Frank. Spatial Ontology: A Geographical Information Point of View, chapter 5, pages 135-153. In Stock [Stock 1997], 1997.

[Freksa and Mark 1999] C. Freksa and D. M. Mark, editors. Spatial Information Theory, Cognitive and Computational Foundations of Geographic Information Science, LNCS 1661. Springer, 1999. International Conference COSIT'99, Stade, Germany, August 1999.

[Ganter and Wille 1999] B. Ganter and R. Wille. Formal Concept Analysis. Springer, Berlin, 1999.

[Godin and Missaoui 1994] R. Godin and R. Missaoui. An incremental concept formation approach for learning from databases. Theoretical Computer Science, 133(2):387-419, 1994.

[Godin et al. 1995] R. Godin, R. Missaoui, and H. Alaoui. Incremental Concept Formation Algorithms Based on Galois (Concepts) Lattices. Computational Intelligence, 11(2):246$267,1995$.

[Grigni et al.1995] M. Grigni, D. Papadias, and C. Papadimitriou. Topological inference. In Proceedings of the 14th IJCAI, Montréal, Canada, pages 901-906, 1995.

[Guénoche and Van Mechelen 1993] A. Guénoche and I. Van Mechelen. Galois Approach to the Induction of Concepts. In I. Van Mechelen, J. Hampton, R.S. Michalski, and P. Theuns, editors, Categories and Concepts. Theoretical Views and Inductive Data Analysis, pages 287-308. Academic Press, London, 1993. 
[Haarslev et al. 1998] V. Haarslev, C. Lutz, and R. Möller. Foundations of Spatioterminological Reasoning with Description Logics. In Proceedings of the Sixth International Conference on Principles of Knowledge Representation and Reasoning KR'98, pages 112123, Trento, Italia, june 1998. Morgan Kaufmann.

[Haarslev et al. 1999] V. Haarslev, C. Lutz, and R. Möller. A description logic with concrete domains and a role-forming predicate operator. Journal of Logic and Computation, $9(3): 351-384,1999$.

[Kawashima et al. 1994] T. Kawashima, K. Yoshino, and Y. Aoki. Qualitative Image Analysis of Group Behaviour. In IEEE Computer Vision and Pattern Recognition (CVPR'94), pages 690-693, 1994.

[Kong and Rosenfeld 1989] T. Y. Kong and A. Rosenfeld. Digital topology: introduction and survey. Computer Vision, Graphics, and Image Processing, 48:357-393, 1989.

[Kovalevsky 1989] V. A. Kovalevsky. Finite topology as applied to image analysis. Computer Vision, Graphics, and Image Processing, 46:141-161, 1989.

[Latecki et al. 1995] L. Latecki, U. Eckhardt, and A. Rozenfeld. Well-composed sets. Computer Vision and Image Understanding, 61(1):70-83, January 1995.

[Le Ber and Mangelinck 1998] F. Le Ber and L. Mangelinck. A formal representation of landscape spatial patterns to analyze satellite images. AI Applications, 12(1-3):51-59, 1998 .

[Le Ber et al. 1999] F. Le Ber, L. Mangelinck, and A. Napoli. Représentation de relations et classification de structures spatiales. RIA, 13(2):441-467, décembre 1999.

[Lesniewski 1927 1931] S. Lesniewski. O podstawach matematyki (On the foundations of mathematics). Przeglad filozoficzny (Philosophical Review), 30-34, 1927-1931.

[Lieber and Napoli 1996] J. Lieber and A. Napoli. Using Classification in Case-Based Planning. In W. Wahlster, editor, Proceedings of the 12th European Conference on Artificial Intelligence (ECAI-96), Budapest, Hungary, pages 132-136. John Wiley \& Sons Ltd., 1996.

[Lieber and Napoli 1998] J. Lieber and A. Napoli. Correct and Complete Retrieval for CaseBased Problem-Solving. In H. Prade, editor, Proceedings of the 13th European Conference on Artificial Intelligence (ECAI'98), Brighton, UK, pages 68-72. John Wiley \& Sons Ltd., 1998.

[Ligozat 1999] G. Ligozat. Simple Models for Simple Calculi. In Freksa and Mark [Freksa and Mark 1999], pages 173-188. International Conference COSIT'99, Stade, Germany, August 1999. 
Le Ber, Mangelinck \& Napoli

[MacGregor and Brill 1992] R.M. MacGregor and D. Brill. Recognition Algorithms for the Loom Classifier. In Proceedings of AAAI'92, San Jose, California, pages 774-779, 1992.

[MacGregor 1991] R. M. MacGregor. Inside the LOOM Description Classifier. ACM Sigart Bulletin, 2(3):88-92, 1991.

[Mangelinck et al. 1996] L. Mangelinck, F. Le Ber, and S. Tabbone. Étude pour la reconnaissance de paysages agricoles sur des images satellitaires. In 10ième Congrès Reconnaissance de Formes et Intelligence Artificielle, pages 53-59. AFCET, janvier 1996. Rennes, France.

[Mangelinck 1998] L. Mangelinck. Représentation et classification de structures spatiales. Application à la reconnaissance de paysages agricoles. Thèse de doctorat, Université Henri Poincaré Nancy 1, octobre 1998.

[Masini et al. 1991] G. Masini, A. Napoli, D. Colnet, D. Léonard, and K. Tombre. ObjectOriented Languages. Academic Press, London, 1991.

[Masolo and Vieu 1999] C. Masolo and L. Vieu. Atomicity vs. Infinite Divisibility of Space. In Freksa and Mark [Freksa and Mark 1999], pages 235-250. International Conference COSIT'99, Stade, Germany, August 1999.

[Masolo 2000] C. Masolo. Critères de comparaison et de construction de théories axiomatiques pour la représentation de connaissances : ontologies de l'espace et du temps. Thèse de doctorat, Université Paul Sabatier - Toulouse et Università degli Studi di Padova, février 2000 .

[Missikoff and Scholl 1989] M. Missikoff and M. Scholl. An Algorithm for Insertion into a Lattice: Application to Type Classification. In W. Litwin and H.-J. Scheck, editors, Foundations of Data Organization and Algorithms, LNCS 367, pages 64-82. SpringerVerlag, Berlin, 1989.

[Möller and Wessel 1999] R. Möller and M. Wessel. Terminological default reasoning about spatial information: A first step. In Freksa and Mark [Freksa and Mark 1999], pages 189-204. International Conference COSIT'99, Stade, Germany, August 1999.

[Napoli et al.1994] A. Napoli, C. Laurenço, and R. Ducournau. An object-based representation system for organic synthesis planning. International Journal of Human-Computer Studies, 41(1/2):5-32, 1994.

[Napoli 1992] A. Napoli. Subsumption and Classification-Based Reasoning in Object-Based Representations. In Proceedings of the 10th European Conference on Artificial Intelligence (ECAI'92), Vienna, Austria, pages 425-429, 1992.

[Nebel 2000] B. Nebel. Knowledge representation and reasoning - the theoretical side of ai. In W. Horn, editor, 14th European Conference on Artificial Intelligence - ECAI 2000 Proceedings, page 763, 2000. 
[Price et al. 1994] K. Price, T. Russ, and R. MacGregor. Knowledge Representation for Computer Vision: The VEIL Project. In ARPA Image Understanding Workshop, 1994.

[Randell and Cohn 1992] D. A. Randell and A. G. Cohn. Exploiting Lattices in a Theory of Space and Time. Computers Math. Applic., 23(6-9):459-476, 1992.

[Randell et al.1992a] D. A. Randell, A. G. Cohn, and Z. Cui. Computing Transitivity Tables: A Challenge For Automated Theorem Provers. In Proceedings of the 11th International Conference on Automated DEduction, CADE'92, LNCS 607. Springer-Verlag, 1992.

[Randell et al.1992b] D. A. Randell, Z. Cui, and A. G. Cohn. A Spatial Logic based on Regions and Connection. In 3rd International Conference on Knowledge Representation and Reasoning, pages 165-176. Morgan Kaufmann, 1992.

[Renz and Nebel 1999] J. Renz and B. Nebel. On the complexity of qualitative spatial reasoning: A maximal tractable fragment of the region connection calculus. Artificial Intelligence, 108(1-2):69-123, 1999.

[Renz 1998] J. Renz. A canonical model of the region connection calculus. In T. Cohn, L. Schubert, and S. Shapiro, editors, Proceedings of the Sixth International Conference on Principles of Knowledge Representation and Reasoning (KR'98), Trento, Italy, pages 330-341, 1998.

[Renz 1999] J. Renz. Maximal tractable fragment of the region connection calculus: a complete analysis. In Proceedings of the 16th IJCAI, Stockholm, Sweden, pages 448-454, 1999.

[Russ et al. 1996] T. Russ, R. MacGregor, B. Salemi, K. Price, and R. Nevatia. VEIL: Combining Semantic Knowledge with Image Understanding. In ARPA Image Understanding Workshop, 1996.

[Simon and Napoli 1999] A. Simon and A. Napoli. Building viewpoints in an object-based representation system for knowledge discovery in databases. In S. Rubin, editor, Proceedings of the First International Conference on Information Reuse and Integration (IRI'99), Atlanta, Geogia, pages 104-108. The International Society for Computers and Their Applications, ISCA, 1999.

[Stock 1997] O. Stock, editor. Spatial and Temporal Reasoning. Kluwer Academic Publishers, 1997.

[Varzi 1996] A. C. Varzi. Parts, wholes, and part-whole relations : the prospects of mereotopology. Data \& Knowledge Engineering, 20:259-286, 1996.

[Varzi 1998] A. C. Varzi. Basic problems of mereotopology. In N. Guarino, editor, Formal Ontology in information systems, (FOIS'98), pages 29-38, Trento, Italy, June 6-8 1998. IOS Press. 
[Vieu 1993] L. Vieu. A Logical Framework for Reasoning about Space. In Spatial Information Theory, COSIT'93, LNCS 716, pages 25-35. Springer-Verlag, 1993.

[Vieu 1997] L. Vieu. Spatial and Temporal Reasoning, chapter 1: Spatial Representation and Reasoning in Artificial Intelligence. In Stock [Stock 1997], 1997.

[Wille 1992] R. Wille. Concept lattices and conceptual knowledge systems. Computers $\mathscr{E}$ Mathematics With Applications, 23(6-9):493-515, 1992.

[Winston et al.1987] M. E. Winston, R. Chaffin, and D. Hermann. A taxonomy of partwhole relations. Cognitive Science, 11:417-444, 1987.

[Winter and Frank 1999] S. Winter and A. U. Frank. Functional Extensions of a Raster Representation for Topological Relations. In Interoperating Geographic Informations Systems, 2nd Int. Conference, INTEROP'99, LNCS 1580, pages 293-304, Zürich, 1999. Springer. 


\section{Contents}

1 Related work 5

1.1 Topological relations: theories . . . . . . . . . . . . . . . . 5

1.2 Computation of topological relations . . . . . . . . . . . . . 7

1.3 Mathematical properties of topological relations . . . . . . . . . . . . . 9

1.4 Some applications in connection with topological relations . . . . . . . . . . . 10

2 Computing topological relations on raster images 12

2.1 The boundary problem . . . . . . . . . . . . . . . . . . . . . 12

2.2 Computational operations for defining topological relations . . . . . . . . 14

3 Two lattices of topological relations $\quad \mathbf{1 5}$

3.1 A lattice based on the subsets of $\mathcal{B} \ldots \ldots \ldots \ldots \ldots \ldots$

3.2 A smooth introduction to the Galois theorv and formal concept analvsis . . . 18

3.3 A Galois lattice based on topological relations . . . . . . . . . . . . . . . . 19

3.3.1 The design of the lattice . . . . . . . . . . . . . . . . . . . 19

3.3.2 The lattice ordering and the implication between relations . . . . . . . 22

3.4 Properties of the $\mathcal{T}_{c}$ lattice . . . . . . . . . . . . . . . 22

3.4.1 The characteristics of the elements of $\mathcal{T}_{d} \ldots \ldots \ldots . \ldots 22$

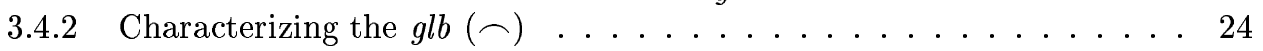

3.4.3 The minimal set of conditions for checking a relation . . . . . . . . 24

3.4.4 Characterizing the $l u b(\smile) \ldots \ldots \ldots \ldots \ldots \ldots$

4 Elements for the comparison of the lattices 25

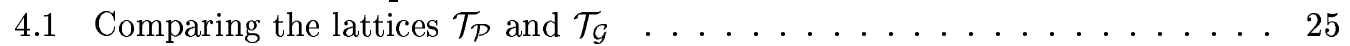

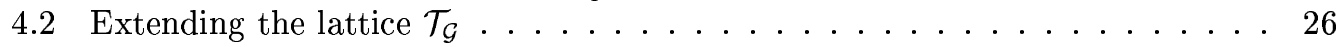

4.2.1 The design of the extended lattice $\mathcal{T}_{a s} \ldots \ldots \ldots$. . . . . . . 26

4.2 .2 Comparing $\mathcal{T}_{c}$ and $\mathcal{T}_{c s} \ldots \ldots \ldots \ldots \ldots . \ldots \ldots 28$

4.3 A second Galois lattice . . . . . . . . . . . . . . . . . . . . 29

4.3.1 The design of the lattice . . . . . . . . . . . . . . . . . . . 29

4.3.2 Comparing the Galois lattices $\mathcal{T}_{\mathcal{c}}$ and $\mathcal{T}_{c} \ldots \ldots \ldots$

4.4 Reasoning with lattices of topological relations . . . . . . . . . . . . . . 32

$5 \quad$ Lattice-based classification of spatial relations and structures $\quad 33$

5.1 Inferences on the relations . . . . . . . . . . . . . . . . 33

5.2 The computation of the relations $\ldots \ldots \ldots \ldots \ldots \ldots$

5.3 Classification of spatial structures . . . . . . . . . . . . . . 36

5.4 Related work $\ldots \ldots \ldots \ldots \ldots \ldots \ldots$

$\begin{array}{lll}6 & \text { Evaluation and research perspectives } & 39\end{array}$ 


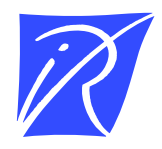

Unité de recherche INRIA Lorraine

LORIA, Technopôle de Nancy-Brabois - Campus scientifique 615, rue du Jardin Botanique - BP 101 - 54602 Villers-lès-Nancy Cedex (France)

Unité de recherche INRIA Rennes : IRISA, Campus universitaire de Beaulieu - 35042 Rennes Cedex (France)

Unité de recherche INRIA Rhône-Alpes : 655, avenue de l'Europe - 38330 Montbonnot-St-Martin (France)

Unité de recherche INRIA Rocquencourt : Domaine de Voluceau - Rocquencourt - BP 105 - 78153 Le Chesnay Cedex (France)

Unité de recherche INRIA Sophia Antipolis : 2004, route des Lucioles - BP 93 - 06902 Sophia Antipolis Cedex (France) 